\title{
The family Phoxocephalopsidae (Crustacea: Amphipoda) from Brazilian coastal waters with description of four new species
}

\author{
PRISCILA S. DO NASCIMENTO AND CRISTIANA S. SEREJO \\ Museu Nacional, Universidade Federal do Rio de Janeiro/UFRJ, Departamento de Invertebrados, Setor \\ de Carcinologia, Quinta da Boa Vista, s/n, São Cristóvão, 20940-040 Rio de Janeiro, RJ, Brazil
}

Manuscript received on February 10, 2018; accepted for publication on June 3, 2018

\begin{abstract}
Phoxocephalopsids are fossorial amphipods endemic to the Southern Hemisphere and currently encompass five genera and 15 species, including the four new species herein described. Previous records of the family along the Brazilian coast are limited to Phoxocephalopsis zimmeri Schellenberg, 1931. Based on material from the Crustacean Collection of Museu Nacional/UFRJ four new species of Phoxocephalopsidae are herein described: Phoxocephalopsis ruffoi sp. nov. from Rio de Janeiro, São Paulo, Paraná and Rio Grande do Sul $\left(23^{\circ}-30^{\circ} \mathrm{S}\right)$; Puelche irenae sp. nov. from Espírito Santo and Rio de Janeiro $\left(19^{\circ}-23^{\circ} \mathrm{S}\right)$; Puelche longidactylus sp. nov. from Bahia, Espírito Santo and Rio de Janeiro $\left(12^{\circ}-23^{\circ} \mathrm{S}\right)$ and Puelche mourae sp. nov. found only at Rio de Janeiro. High diversity was recorded from the Campos Basin region, which extends from the north of Rio de Janeiro to south of Espírito Santo $\left(21^{\circ}-23^{\circ} \mathrm{S}\right)$, with the presence of all four phoxocephalid species. A key to Phoxocephalopsidae species from Brazil, including P. orensanzi Barnard and Clark, 1982 is provided.
\end{abstract}

Key words: southwestern Atlantic, Campos Basin, new species, Phoxocephalopsis, Puelche, taxonomy.

\section{INTRODUCTION}

The family Phoxocephalopsidae Barnard and Drummond, 1982 is endemic to the Southern Hemisphere and includes amphipods ranging from 5 to $15 \mathrm{~mm}$ in length that live within unconsolidated substrates. Barnard and Drummond (1982) created the family Phoxocephalopsidae as part of the superfamily Haustorioidea, together with fossorial families as Condukiidae Barnard and Drummond, 1982; Haustoriidae Stebbing, 1906; Phoxocephalidae G.O. Sars, 1891;

Correspondence to: Priscila Soares do Nascimento

E-mail: nascimentops23@gmail.com
Platyischnopidae Barnard and Drummond, 1979; Urohaustoriidae Barnard and Drummond, 1982; Urothoidae Bousfield, 1978; and Zobrachoidae Barnard and Drummond, 1982. This classification was questioned by Bousfield and Shih (1994) because all these families were endemic to the Southern Hemisphere, except Haustoriidae, which inhabits coastal waters of the Northern Hemisphere. Thus, Bousfield and Shih (1994) established the superfamily Phoxocephaloidea, including only southern Hemisphere families, without the Haustoriidae. More recently, Lowry and Myers (2017) re-estabilished the superfamily Haustorioideabased on a phylogenetic analysis, with 
all families included by Barnard and Drummond (1982), including also other fossorial families as Cheidae Thurston, 1982; Ipanemidae Barnard and Thomas, 1988; Otagiidae Hughes and Lörz, 2013; Pontoporeiidae Dana, 1852; Priscillinidae d'Udekem and d'Acoz, 2006; Sinurothoidae Ren, 1999.

The cold water genus Phoxocephalopsis Schellenberg, 1931 was treated as the type genus of the family erected by Barnard and Drummond (1982). Puelche Barnard and Clark, 1982 was described from Argentina, a monotypic genus until the present study and Eophoxocephalopsis Thurston, 1989 from the Falkland Islands. More recently, Sittrop et al. (2014) moved Pseudurothoe Ledoyer, 1986 and Urothopsis Ledoyer, 1967 from Urothoidae to Phoxocephalopsidae, based on the analysis of the respective type species of each genus. Therefore, the family Phoxocephalopsidae comprises five genera and 15 species, including the new species described herein: Phoxocephalopsis (6 spp.), Puelche (4 spp.), Eophoxocephalopsis (3 spp.), Pseudurothoe (1 spp.) and Urothopsis (1 spp.).

The distribution of Phoxocephalopsis, Puelche and Eophoxocephalopsis is restricted to the South American coast, including Brazil, Uruguay, Argentina, Falklands Islands, Chile and the Magellan Region. Pseudurothoe and Urothopsis are found in the South Africa and Madagascar, respectively.

In Brazil, only Phoxocephalopsis zimmeri Schellenberg, 1931 was previously recorded from São Paulo state (Wakabara et al. 1991, Wakabara and Serejo 1998). Ruffo (1956) worked with material from Santos, SP, which was identified as P. zimmeri. However, Ruffo (1956) presented the dactylus of pereopod five in $P$. zimmeri with groups of long robust setae at the inner edge, differing from the original description of Schellenberg (1931). Further works referred to Ruffo's material as a different species (Barnard and Clark 1984,
Thurston 1989, Barnard and Karaman 1991), but no formal species description was provided. The present study fully describes Phoxocephalopsis ruffoi $\mathrm{sp}$. nov. based on material from the Campos Basin, RJ, with additional records for the states of Paraná and Rio Grande do Sul. Material from the states of Rio de Janeiro and Espírito Santo have been examined yielding new information on the distribution of $P$. zimmeri. In addition, samples from Campos Basin, RJ allowed the description of three new species for the genus Puelche, with records for the states of Bahia and Espírito Santo.

\section{MATERIALS AND METHODS}

Most of the material examined was collected as part of the three projects as follows: 1. Rapid Assessment of Biodiversity - Ilha Grande Bay Program (2003) with summer sampling (from October to December) using a manual corer of 100 $\mathrm{mm}$ in diameter by $150 \mathrm{~mm}$ in height between depths of 80 and $100 \mathrm{~m}$. The study area was Ilha Grande Bay $\left(22^{\circ} 50^{\prime}-23^{\circ} 20^{\prime} \mathrm{S}, 44^{\circ} 00^{\prime}-44^{\circ} 45^{\prime} \mathrm{W}\right)$, south of Rio de Janeiro state, Brazil (Creed et al. 2007); 2. HABITATS Project - Environmental Heterogeneity of the Campos Basin (2008 to 2009) with samples collected from the continental shelf at depths of 25 to $150 \mathrm{~m}$,from January to March (summer - rainy period) and from May to July (winter- dry period), using a Van Veen grab $92 \times 80 \times 40 \mathrm{~cm}$ (RibeiroFerreira et al. 2017); 3. AMBES Project (2010 to 2013) - Marine environmental characterization of the Espírito Santo Basin (2010 to 2013) with samples collected on the Continental shelf from depths of 12 to $150 \mathrm{~m}$, from December to February (summer) and from June to July (winter) using Van Veen grab $92 \times 80 \times 40 \mathrm{~cm}$ and Box Corer gears $50 \times 50 \times 50 \mathrm{~cm}$. The two later projects were coordinated by CENPES/PETROBRAS (Petróleo Brasileiro S.A.) as part of the characterization of the Campos Basin and Espírito Santo Basin. Detail information on the sediment cover, bathymetry and 
oceanographic water mass of the Campos Basin region is available in Falcão et al. (2017).

Phoxocephalopsidade specimens from studies of spatial distribution of benthic macrofauna in beaches of Rio Grande do Sul state was also analyzed. The study areas were the beaches of Tramandaí $\left(30^{\circ} 00^{\prime} 22^{\prime \prime} \mathrm{S}-50^{\circ} 07^{\prime} 821^{\prime \prime} \mathrm{W}\right)$, Harmonia $\left(29^{\circ} 54^{\prime} 857^{\prime \prime} \mathrm{S}-50^{\circ} 05^{\prime} 421^{\prime \prime} \mathrm{W}\right)$ and Jardim do Éden $\left(30^{\circ} 05^{\prime} 800^{\prime}\right.$ ' S - 50 10 ' $238^{\circ}$ " W), with sampling in April of 2003 using a PVC extractor tube with $20 \mathrm{~cm}$ diameter over transects set on each beach, from the intertidal zone down to $1.0 \mathrm{~m}$ depth seawards (Neves and Bemvenuti 2006, 2009).

There were also analyzed samples deposited in the Crustacea Collection of the Museu Nacional from the Universidade Federal do Rio de Janeiro (MNRJ) by different collectors from the states of Bahia, Rio de Janeiro and Paraná using manual corers (from 1 to $22 \mathrm{~m}$ of depth).

All samples were examined and identified under a Zeiss Stemi SV6 stereomicroscope and ZEISS Axioscope coupled with a camera lucida. Specimens were dissected and mounted in semipermanent slides made with glycerin jelly. Illustrations were prepared using Inkscape 0.48 graphics program with the aid of a Wacom Intuos CTL480L pen table adapted from the methodology proposed by Coleman (2006).

There were also produced photographs on stereoscopic microscope ZEISS Discovery V.20 with coupled camera and image processing in extended depth of focus mode by ZEN Pro 2 (Blue edition) program to illustrate the habitus of Puelche species.

General setae nomenclature follows Zimmer et al. (2009). However, Zimmer et al. (2009) treated penicillate setae, broadly used in papers dealing with fossorial amphipods, as type of pappose seta. The present work will consider the penicillate setae as a distinct type of seta, characterized by a short shaft, with only distal portion of shaft branched in long setules, forming a tuft (Fig. 5d). The term "pappose setae" will be used for long shaft setae, at least half the length of shaft branched in setules, not forming a tuft. The term "robust setae" will be used (sensu Watling 1989), instead of "cuspidate setae" from Zimmer et al. (2009). All material herein examined are deposited in the Crustacea Collection of the Museu Nacional from the Universidade Federal do Rio de Janeiro (MNRJ).

Abbreviations in the figures: $\mathrm{A}=$ antenna; $\mathrm{D}=$ dactylus; FA = flagellum antenna; $\mathrm{G}=$ gnathopod; $\mathrm{Hd}=$ head; $\mathrm{Hb}=$ habitus; $\mathrm{LL}=$ lower lip; $\mathrm{LMl}=$ left lacinia mobilis; $\mathrm{LMr}=$ right lacinia mobilis; $\mathrm{Mdl}=$ left mandible; $\mathrm{Mdr}=$ right mandible; $\mathrm{Mx}=$ maxilla; Mxp = maxilliped; $\mathrm{Pln}=$ pleon; $\mathrm{P}=$ pereopod; $\mathrm{R}=$ raker; Palp $\mathrm{r}=$ right palp; $\mathrm{T}=$ telson; $\mathrm{U}=$ uropod; $\mathrm{UL}=$ upper lip.

\section{TAXONOMY}

Order Amphipoda Latreille, 1816

Suborder Amphilochidea Boeck, 1871 (Lowry and Myers, 2017)

Infraorder Lysianassida Dana, 1849 (Lowry and Myers, 2017)

Parvorder Haustoriidira Stebbing, 1906 (Lowry and Myers, 2017)

Superfamily Haustorioidea Stebbing, 1906 (Barnard and Drummond, 1982)

Family Phoxocephalopsidae Barnard and Drummond, 1982

Phoxocephalopsis ruffoi sp. nov.

Figures 1-4

Phoxocephalopsis zimmeri. — Ruffo, 1956: 115118, fig. I (1-4).

? Phoxocephalopsis sp. - Barnard and Clark, 1984: 88 (key). — Thurston, 1989: 309 (key).

ZooBank Life Science Identifier (LSID) urn:1sid:zoobank.org:act:D4145AEA-3FD0-49EE91F1-402D945EEBDE 


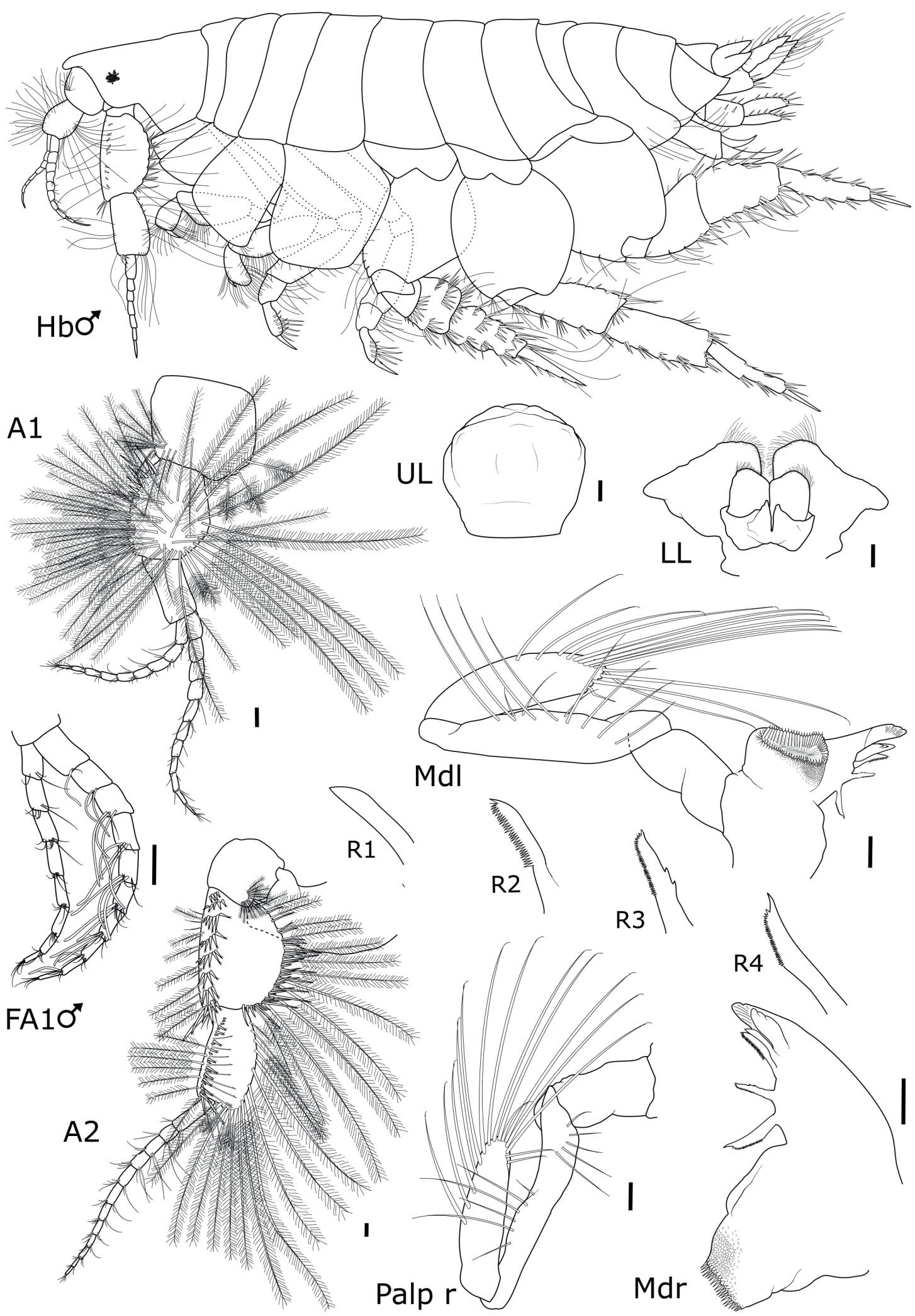

Figure 1 - Phoxocephalopsis ruffoi sp. nov. Female, 7.5 mm, MNRJ 22101: Antennae and mouthparts. Male, 6.9 $\mathrm{mm}$, MNRJ 28745: Habitus and flagellum of antenna 1 . Scales $=0.1 \mathrm{~mm}$. 


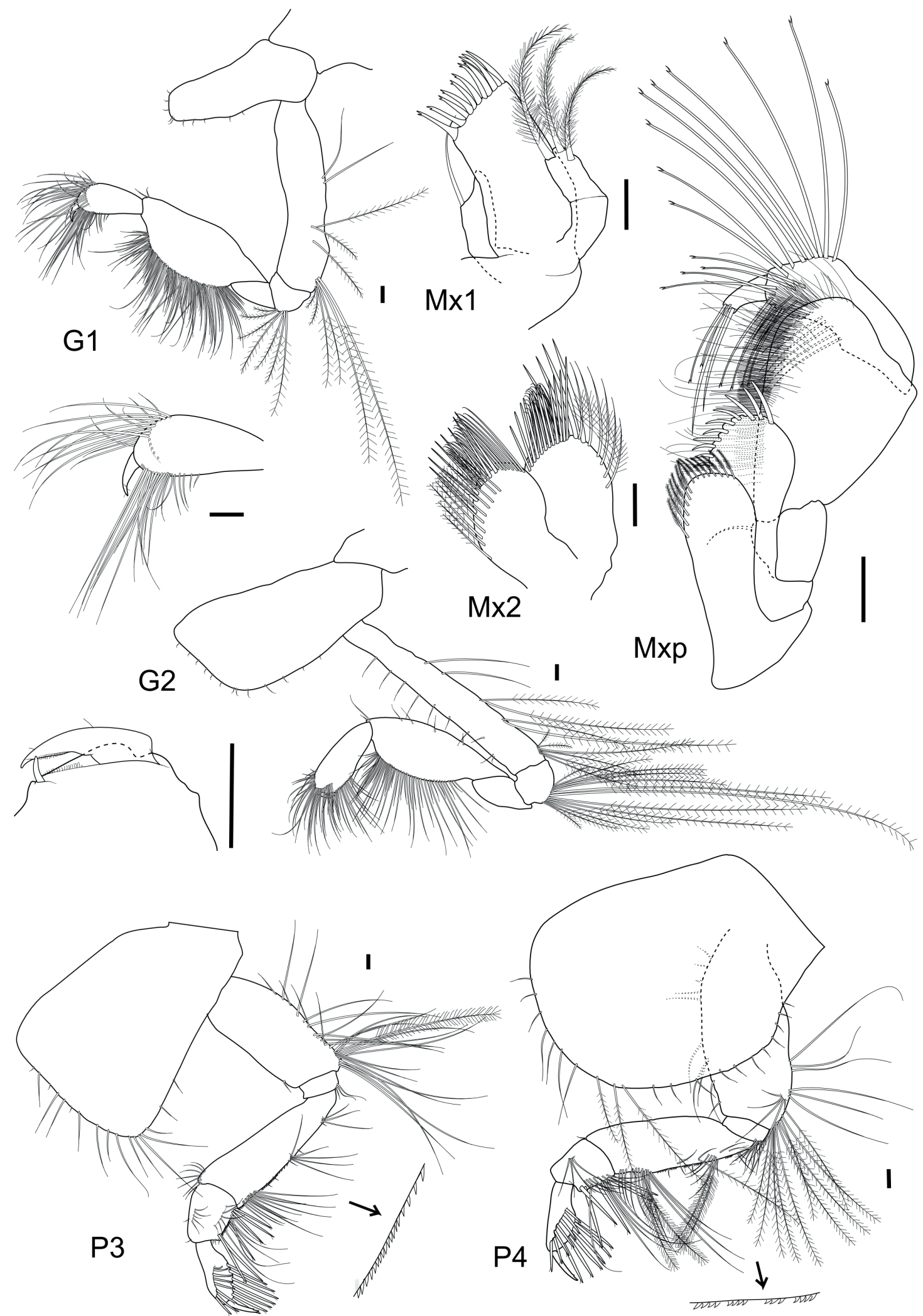

Figure 2 - Phoxocephalopsis ruffoi sp. nov. Female, 7.5 mm, MNRJ 22101: Mouthparts, gnathopods and pereopods 3 - 4. Scales $=0.1 \mathrm{~mm}$. 


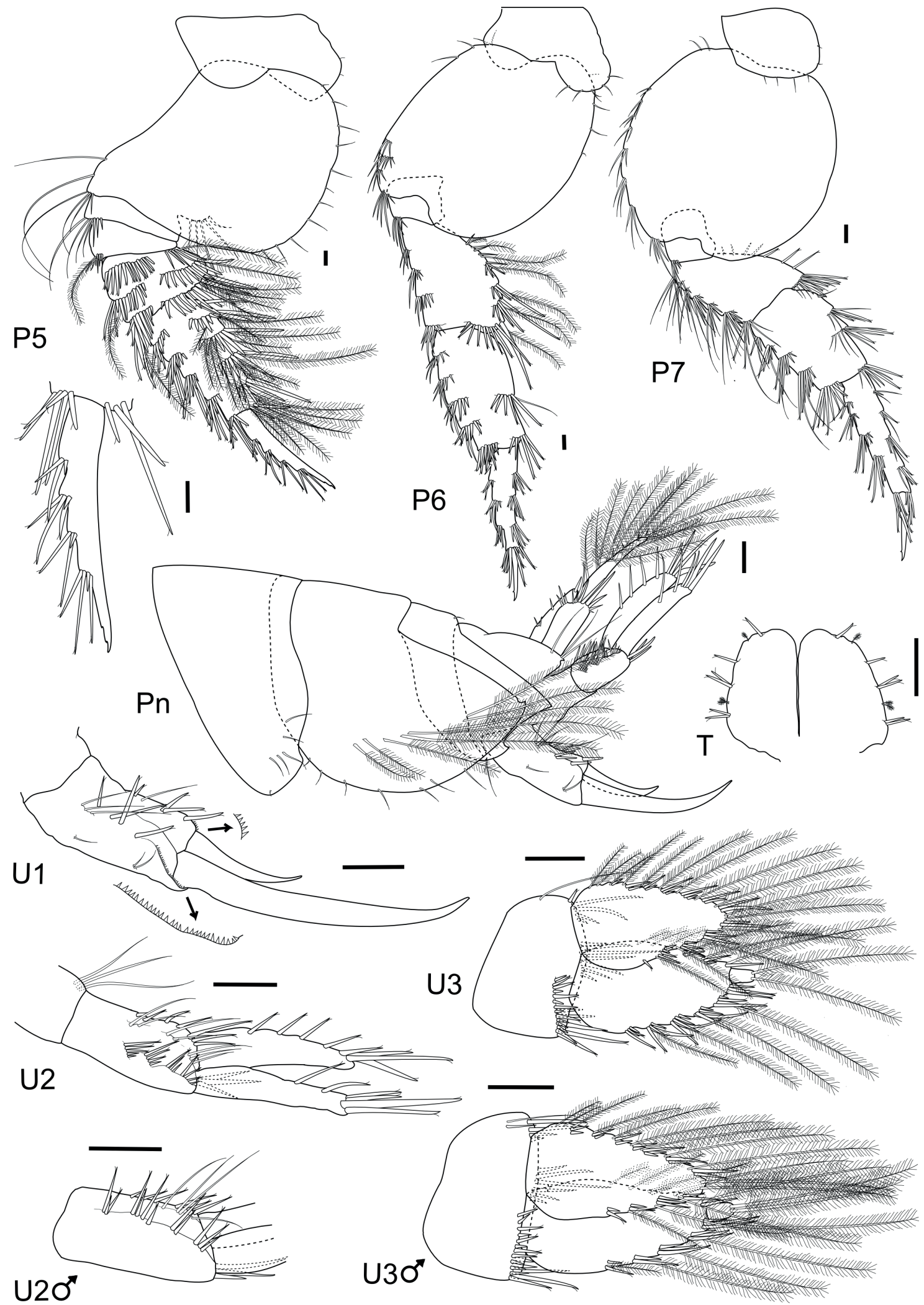

Figure 3 - Phoxocephalopsis ruffoi sp. nov. Female, 7.5 mm, MNRJ 22101: Pereopods 5 - 7, pleon, uropods and telson. Male, $6.9 \mathrm{~mm}$, MNRJ 28745: Uropods $2-3$. Scales $=0.1 \mathrm{~mm}$. 

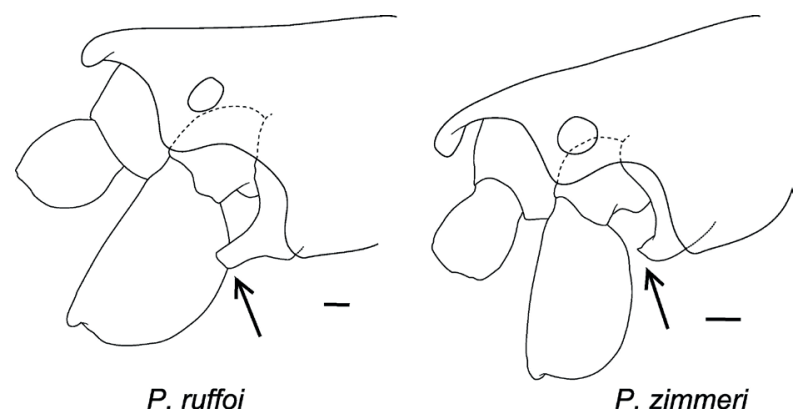

Figure 4 - Phoxocephalopsis ruffoi sp. nov. Female, $7.5 \mathrm{~mm}$, MNRJ 22101, ensiform process truncate. Phoxocephalopsis zimmeri, Female, $7.0 \mathrm{~mm}$, MNRJ 28874, ensiform process acute.

Holotype: Female, length $7.5 \mathrm{~mm}$, Campos Basin, Rio de Janeiro, 215'25.300'’S 4049'12.327”' W, 20 m, MNRJ 22101.

Allotype: Male, length $6.9 \mathrm{~mm}$, Campos Basin, Rio de Janeiro, 21\%43'22.256”'S 40³1'52.220” W, 26 m, MNRJ 28745.

Paratypes: Rio de Janeiro - 2 females, 22 58'2.28'S 426'57.14”'W, 10 m, MNRJ 10557;

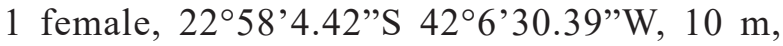
MNRJ 10564; 1 female, 1 juvenile, 21 $1^{\circ} 55^{\prime} 30.810^{\prime \prime} \mathrm{S}$ 4043'22.824'”, 20 m, MNRJ 13949; 15 males, 16 females, 5 juveniles, $23^{\circ} 4^{\prime} 42.68^{\prime \prime}$ 'S 4347'39.78' W, $2 \mathrm{~m}$, MNRJ 14058; 1 male, 2150'20.765"S 40³1'38.459'W, 28 m, MNRJ 18594; 3 females, $21^{\circ} 45^{\prime} 2.327^{\prime \prime S} 40^{\circ} 25^{\prime} 45.451$ 'W, 27 m, MNRJ 19050; 2 males, 2150'20.315"S 40³1'38.915"W, 27 m, MNRJ 22096; 1 female, 21 45'2.154"'S 40²5'44.878' 'W, 26 m, MNRJ 22098; 1 female, $21^{\circ} 55^{\prime} 18.410^{\prime \prime} \mathrm{S} 40^{\circ} 55^{\prime} 1.734$ '”, $17 \mathrm{~m}$, MNRJ 22099; 1 female, 1 juvenile, 21 ${ }^{\circ} 55^{\prime} 32.047^{\prime \prime} \mathrm{S}$ 4043'24.119'"W, 20 m, MNRJ 22100; 1 female, $21^{\circ} 49^{\prime} 58.813^{\prime \prime} \mathrm{S} 40^{\circ} 49^{\prime} 2.818^{\prime \prime} \mathrm{W}, 23 \mathrm{~m}, \mathrm{MNRJ}$ 28862; 1 male, 2134'39.794"'S 40³5'17.09'W, 23 m, MNRJ 28697; 1 female, 21³9'59.987"S 40³4’34.120”W, 23 m, MNRJ 28698. Paraná - 1 male, 1 female, 1 juvenile, $25^{\circ} 36^{\prime} 45.53^{\prime \prime} \mathrm{S}$ 48 22'53.85"W, MNRJ 28696. Rio Grande do Sul - 2 females, 1 juvenile, $30^{\circ} 05^{\prime} 8.00$ " $\mathrm{S}$ $50^{\circ} 10^{\prime} 2.38^{\prime \prime} \mathrm{W}, 1 \mathrm{~m}, \mathrm{MNRJ} 28861 ; 2$ females,
29 54'8.57"S 5005'4.21'”, 1 m, MNRJ 28863; 1 male, 2954'8.57'S - 5005'4.21'”W, 1 m, MNRJ 28864 .

Etymology: Named in honour of Dr. Sandro Ruffo (Museo Civico di Storia Naturale, Verona, Italy), one of the great amphipodologists of our time and who first noticed the main difference between the new species collected in Santos, SP and Phoxocephalopsis zimmeri.

Diagnosis: Antenna 2, article 2 with ensiform process truncate apically. Incisors toothed; rakers present, 4 on the right mandible and 3 on the left mandible. Maxilla 1, inner plate with 1 medial simple seta; outer plate, weakly 2 -articulated, with 3 pappose setae apically. Pereopod 5, dactylus anterior margin notched with groups of robust setae. Epimeron 2 dominant. Epimeron 3 poorly setose with small ventral tooth and posteroventral angle acute. Uropod 2, ramus with dorsal and apical setae.

Description: Female, $7.5 \mathrm{~mm}$, MNRJ 22101. Head $1.3 \mathrm{x}$ longer than wide, rostrum about $1 / 5$ of the length of head, ventral margin medially excavated, forming a "cheek", small eyes.

Antenna 1, articles 1-2 of peduncle broad and subequal in length, article 3 thinner and length $0.6 \mathrm{x}$ length of article 2; article 1 anterolateral armament with plumose setae, posterior margin with 3 penicillate setae; article 2 strongly setose with many plumose setae, anterior margin with 8 robust setae, posterodistal angle with 2 penicillate setae; article 3 with 1 plumose seta on posterodistal angle; primary flagellum with 11 articles; accessory flagellum with 9 articles. Antenna 2, article 2 with ensiform process truncate apically; article 3 with curve row of short plumose setae; articles 4-5 anterior and posterior margins with plumose setae; article 4 broad, anterior margin with short simple setae and groups of 4-5 robust setae each with accessory seta (Fig. 5a), posterior margin covered by robust setae; article 5 thinner and $0.8 \times$ length of article 4 , anterior margin with row of robust setae with accessory 
seta interspersed with simple setae with lamellate tip (Fig 5b); flagellum 12-articulated.

Upper lip rounded, as long as wide, naked. Mandible, right incisor with 2 blunt teeth and left with 3; right and left lacinia mobilis with 3 teeth; 4 rakers on the right mandible (Fig. 1 r1-4) and 3 rakers on the left(Fig. 1 r2-4); molar broad, elongate, with scaly margins, strongly triturative both sides; palp 3-articulated in both sides; article 2 the longest with facial setae; article 3 with distal setae with hooklike tip in both sides; article 3 on the right mandible with 9 long setae on outer margin, 1 short seta apically, 3 long setae on inner margin followed by 2 short setae (setal formula 9-1-3-2), article 3 on the left mandible with 9 long setae on outer margin, 1 short seta apically, 3 long setae on inner margin followed by 1 short seta (setal formula 9-1-3-1). Lower lip with mandibular lobes well developed, laterally directed. Maxilla 1, inner plate tapered, reaching about half of length of outer plate, with 1 long medial simple seta; outer plate with 11 robust setae, 8 of them with distal tooth; palp half the length of the outer plate, weakly 2-articulated, with 3 pappose setae apically. Maxilla 2, plates subequal in length and width; inner plate with apical row of simple setae, subapical row of robust setae, facial row of plumose setae; outer plate with apical row of simple setae and 4 long robust setae, subapical row of robust setae, apicolateral row of plumose setae. Maxilliped, inner plate with 14 pappose setae apically; outer plate slightly larger than inner plate, with robust setae on inner margin; article 1 with 1 long simple seta on inner margin; article 2 broad, with dense row of simple setae on inner margin; article 3 thinner with simple setae with lamellate tip; article 4 rod-shaped, apex with 2 robust setae and 2 simple setae with lamellate tip.

Coxae 1-4 progressively larger. Gnathopod 1, coxa small, shoe-shaped with small simple setae; basis and ischium with group of plumose setae on posterodistal angle; carpus with posterior tumescence and densely setose on posterior margin; propodus thinner, with simple setae distally; dactylus simple. Gnathopod 2, coxa subrectangular with small simple setae; basis longer than in G1, $7.5 \mathrm{x}$ longer than wide, with simple setae on anterior margin and groups of plumose setae on posterior margin; ischium with a row of long plumose setae on posterior margin; carpus and propodus with setal armament similar to G1; subchelate, palm transverse, setose, distally delimited by a robust seta, dactylus setose, reaching the end of the palm. Pereopods 3, coxa subrectangular, 1.5x longer than wide with simple setae on ventral margin; basis with long simple setae and 1 plumose seta on posterior margin; merus posterior margin toothed on distal half, with 3 groups of simple setae; carpus posterior margin with 6 robust setae with accessory seta (setal formula 3-1-2) and 2 rows of simple setae laterally; propodus with robust setae with accessory seta, 8 on one side of dactylus and 7 on the other;dactylus with distal tooth. Pereopod 4 , coxa subquadrate, $1.1 \mathrm{x}$ longer than wide with simple setae on the ventral and posterior margins and 3 plumose setae on posteroventral angle; basis with simple setae and plumose setae on posterior margin; merus posterior margin toothed, with groups of simple and plumose setae; carpus with 5 robust setae with accessory seta on posterior margin (setal formula 2-1-2); propodus with robust setae with accessory seta, 7 on one side of dactylus and 6 on the other; dactylus with distal tooth. Coxae 5-6 bilobed, lobes subequal. Pereopod 5, basis with distal simple setae on anterior margin and short simple setae on posterior margin; ischium and merus short, with simple and robust setae on anteroventral angle; carpus and propodus with many groups of robust setae with accessory seta, posterior margin with plumose setae; dactylus anterior margin with 4 great notches with groups of robust setae on each one (setal formula 3-4$3-4)$ and 2 small notches without setae distally. Pereopod 6, merus, carpus and propodus anterior 
and posterior margins with groups of robust setae with accessory seta; merus with 1 plumose seta on each group of posterior margin; dactylus with distal tooth. Pereopod 7 longer than 6 , coxa distally rounded; basis subcircular; merus, carpus and propodus with groups of simple setae and robust setae with accessory seta; dactylus longer than dactylus of pereopod 6 with distal tooth.

Epimeron 1 with a few simple setae, with a blunt posteroventral tooth; epimeron 2 strongly dominat with long plumose setae and a small posteroventral tooth; epimeron 3 poorly setose with small ventral tooth and posteroventral angle acute. Pleopods in urohaustoriid form.

Urosomite 1 with posterior covering the other urosomites, with small posteroventral tooth and 3 simple setae on posterior margin. Uropod 1, inner and outer dorsal margins of peduncle toothed distally, each margin with 1 row of robust setae with accessory seta (setal formula 1-2-1); biramus, rami styliform, naked, sharply pointed, inner ramus about half the length of outer ramus. Uropod 2, inner dorsal margin of peduncle with row of 7 robust setae with accessory seta, medial dorsal margin with 7 simple setae, outer dorsal margin with groups of robust setae with accessory seta (setal formula 5-4-3-4), ventral apex with 3 robust setae with accessory seta on inter-ramus position; rami rodlike, subequal in length, each one with 4 apical robust setae with accessory seta; inner ramus with 4 robust setae with accessory seta on dorsal margin and outer ramus with 2. Uropod 3, peduncle wider than long, with groups of robust setae with accessory seta, 5 setae on inner ventral apex, 6 setae ventrally on inter-ramus position and 13 setae dorsolaterally on outer apex; ramus leaflike, inner ramus 1-articulated, as long as article 1 of outer ramus, inner and outer margins with plumose setae and groups of robust setae with accessory seta, 2 plumose setae apically; outer ramus 2-articluted, article 1 inner and outer margins with plumose setae and groups of robust setae with accessory seta, second article $1 / 6$ of article 1 length, with 2 plumose setae apically. Telson as long as wide, deeply cleft, apex of lobes rounded, outer margins of each lobe with 6 robust setae with accessory seta (setal formula $=2-2-1-1)$ and 3 small penicillate setae, 2 proximal and 1 distal.

Dimorphism: Male, $6.9 \mathrm{~mm}$, MNRJ 28745. In general, less setose than female. Antenna 1, primary flagellum with 10 articles, aesthetascs present, formula $=1-2-3-3-3-2-2-1-1-0$; accessory flagellum with 8 articles. Uropod 2, inner dorsal margin of peduncle with row of 6 robust setae with accessory seta, medial dorsal margin with 4 simple setae, outer dorsal margin with groups of robust setae with accessory seta (setal formula 3-4-2-3). Uropod 3, inner ventral apex of peduncle with 4 robust setae, 6 robust setae ventrally on inter-ramus position like female, outer apex with 16 robust setae dorsolaterally; inner ramus, outer margin with one less group of setae than female; outer ramus, article 1 inner and outer margins each one with one less group of setae than female.

Remarks: Phoxocephalopsis ruffoi sp. nov. presents all the diagnostic features of the genus listed by Barnard and Karaman (1991) as: 1) mandible incisors toothed; 2) mandible rakers present; 3) coxa 2 with more than half surface area of coxa 3 ; 4) epimera with posteroventral points; 5) epimeron 2 dominant. The new species is closely related to Phoxocephalopsis zimmeri Schellenberg, 1931, identified by Ruffo (1956) as the same species despite of main differences in dactylus of pereopod 5. Subsequently, Ruffo's species was treated as a different taxon (Barnard and Clark 1984; Thurston 1989), but no formal description was proposed until now. Phoxocephalopsis ruffoi sp. nov. differs from P. zimmeri in the following characters: 1) antenna 2 , article 2 with ensiform process truncate apically (versus acute process) (Fig. 4); 2) right mandible with 4 rakers (versus 2 rakers); 3 ) maxilla 1, outer plate with 3 pappose setae apically (versus 3 simple setae); 4) pereopod 5, dactylus anterior margin 
notched with four groups of robust setae (versus dactylus styliform, lacking groups of robust setae). Type locality: Campos Basin (215'25.300"S 4049'12.327” W), 20 m, Rio de Janeiro, Brazil.

Distribution: Rio de Janeiro - Campos Basin (17-28 m), Figueira Beach and Monte Alto Beach, Arraial do Cabo (10-20 m), Restinga da Marambaia (2 m); São Paulo - Santos (Ruffo, 1956); Paraná - Atami Beach, Pontal do Sul; Rio Grande do Sul - Jardim do Éden Beach, Tramandaí $(1 \mathrm{~m})$ and Harmonia Beach, Imbé (1 m).

Phoxocephalopsis zimmeri Schellenberg, 1931

Figure 4

Phoxocephalopsis zimmeri Schellenberg 1931: 70-73, fig. 36. - Barnard and Clark 1984: 88-97, figs. 1-5. — Wakabara et al. 1991: 75 (distributional data). - Wakabara and Serejo 1998: 578.

Haustoriella psammophila Barnard 1931: 426.

Material examined: Espírito Santo - 1 specimen, 19³1'30"W 39'36'00'”W, 32 m, MNRJ 16726. Rio de Janeiro - 3 specimens, 22 $58^{\prime} 4.42$ 'S 42 6'30.39”'W, 30 m, MNRJ 10568; 1 specimen, 22 58'4.42”S 426'30.39”'W, 40 m, MNRJ 10569; 2 specimens, $22^{\circ} 58$ '4.42”S 426'30.39”W, 60 m, MNRJ 10570; 2 specimens, 22 $58^{\prime} 4.42$ "S 426'30.39”'W, 30 m, MNRJ 10571; 1 specimen, $22^{\circ} 12^{\prime} 53.045^{\prime}$ 'S 40 51 '12.031', $\mathrm{W}, 52 \mathrm{~m}$, MNRJ 28701; 3 specimens, $22^{\circ} 6$ '56.222'"S $40^{\circ} 38^{\prime} 58.224^{\prime \prime} \mathrm{W}, 53 \mathrm{~m}, \mathrm{MNRJ} 28702 ; 16$

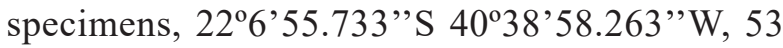

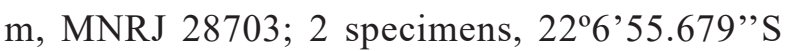
$40^{\circ} 38^{\prime} 58.325^{\prime \prime} \mathrm{W}, 53 \mathrm{~m}, \mathrm{MNRJ} 28704 ; 4$ specimens, $21^{\circ} 43^{\prime} 22.256^{\prime} \mathrm{S} 40^{\circ} 31^{\prime} 52.229^{\prime} \mathrm{W}$, 26 m, MNRJ 28705; 1 specimen, 21 $28^{\circ} 2.517^{\prime \prime} \mathrm{S}$ $40^{\circ} 56^{\prime} 20.614$ ' W, $16 \mathrm{~m}, \mathrm{MNRJ} 28706$; 2 specimens, 21 ${ }^{\circ} 55^{\prime} 18.212^{\prime \prime} \mathrm{S}$ 40 $55^{\circ} 0.708^{\prime \prime} \mathrm{W}, 16$ $\mathrm{m}, \mathrm{MNRJ} 28707 ; 1$ specimen, $22^{\circ} 11{ }^{\prime} 32.065^{\prime \prime} \mathrm{S}$
4055'24.159'’W, 44 m, MNRJ 28708; 1

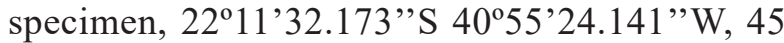
$\mathrm{m}$, MNRJ 28709; 1 specimen, 21 ${ }^{\circ} 55^{\prime} 25.300$ "S 40 49'12.327' 'W, $20 \mathrm{~m}$, MNRJ 28710; 1

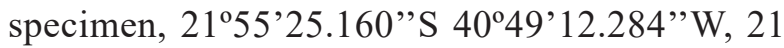
m, MNRJ 28711; 4 specimens, 21 ${ }^{\circ} 55^{\prime} 25.232$ ''S $40^{\circ} 49^{\prime} 12.255^{\prime \prime} \mathrm{W}, 21 \mathrm{~m}, \mathrm{MNRJ} 28712 ; 7$

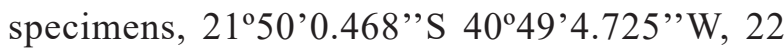
m, MNRJ 28713; 18 specimens, 2133'52.574"S 40 42'53.900'”W, $22 \mathrm{~m}$, MNRJ 28714; 7 specimens, 21'33'54.457''S 4042'55.682'W, 21

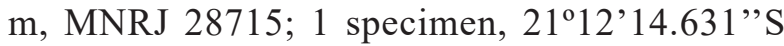
$40^{\circ} 42^{\prime} 26.841^{\prime \prime} \mathrm{W}, 16 \mathrm{~m}, \mathrm{MNRJ} 28716$; 2 specimens, 21 ${ }^{\circ} 44^{\prime} 42.879$ 'S 4043'9.193'W, 22 m, MNRJ 28717; 4 specimens, 2144'42.969' S $40^{\circ} 43^{\prime} 8.347^{\prime} ' \mathrm{~W}, 21 \mathrm{~m}, \mathrm{MNRJ} 28718 ; 6$

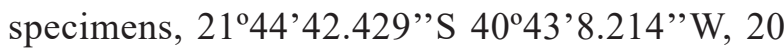
m, MNRJ 28719; 1 specimen, 21 ${ }^{\circ} 55^{\prime} 32.130^{\prime \prime} \mathrm{S}$ 40 43'23.903'”W, 19 m, MNRJ 28720; 1 specimen, 21 ${ }^{\circ} 55^{\prime} 32.047^{\prime}$ 'S 40 43'24.119' W, 20 $\mathrm{m}$, MNRJ 28721; 2 specimens, $22^{\circ} 6^{\prime} 21.254$ ' $\mathrm{S}$ $40^{\circ} 43^{\prime} 39.365^{\prime \prime} \mathrm{W}, 47 \mathrm{~m}$, MNRJ 28722; 5

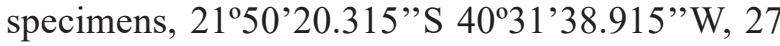
m, MNRJ 28723; 5 specimens, 21 50 '20.906"'S 40³1'39.534'’W, 27 m, MNRJ 28724; 5 specimens, 2150'20.898'S 40³1'39.721'”, 27 m, MNRJ 28725; 2 specimens, 2139'30.521'"S $40^{\circ} 31^{\prime} 27.085^{\prime \prime} \mathrm{W}, 28 \mathrm{~m}, \mathrm{MNRJ} 28726$; 1 specimen, 2139'30.939'"S 40³1'25.652"'W, 28 m, MNRJ 28727; 2 specimens, 2117'51.815'S 40³0'59.224" 'W, 29 m, MNRJ 28728; 4 specimens, $21^{\circ} 45^{\prime} 2.021^{\prime \prime} \mathrm{S} 40^{\circ} 25^{\prime} 45.245^{\prime \prime} \mathrm{W}, 26 \mathrm{~m}$, MNRJ

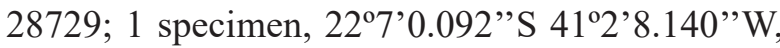
18 m, MNRJ 28730; 1 specimen, 22¹1'30.609' S 40 55'24.468''W, $44 \mathrm{~m}$, MNRJ 28731; 1

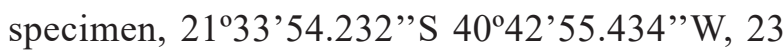
m, MNRJ 28732; 2 specimens, 214'39.982"S $40^{\circ} 43^{\prime} 8.573^{\prime \prime} \mathrm{W}, 21 \mathrm{~m}, \mathrm{MNRJ} 28733$; 1 specimen, $21^{\circ} 44^{\prime} 44.391$ 'S 4043'7.564' $\mathrm{W}, 21$ m, MNRJ 28734; 5 specimens, $21^{\circ} 55^{\prime} 30.810^{\prime \prime} \mathrm{S}$ $40^{\circ} 43^{\prime} 22.824^{\prime \prime} \mathrm{W}, 20 \mathrm{~m}$, MNRJ 28735; 2 specimens, $22^{\circ} 6^{\prime} 22.014$ ' S $40^{\circ} 43^{\prime} 42.326^{\prime \prime} \mathrm{W}, 47$ 
m, MNRJ 28736; 1 specimen, 2150'20.997's 40³1'38.672'’W, $28 \mathrm{~m}$, MNRJ 28737; 1 specimen, $21^{\circ} 17^{\prime} 52.350$ 'S $40^{\circ} 31^{\prime} 0.169^{\prime \prime} \mathrm{W}, 30$ m, MNRJ 28738; 4 specimens, 2134'12.597's $40^{\circ} 25^{\prime} 31.241^{\prime \prime} \mathrm{W}, 29 \mathrm{~m}, \mathrm{MNRJ} 28739 ; 1$

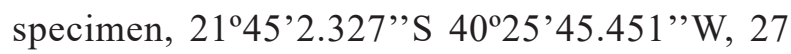
m, MNRJ 28740; 1 specimen, 21 $41^{\circ} 1.967$ 'S $40^{\circ} 25^{\prime} 45.211^{\prime \prime} \mathrm{W}, 26 \mathrm{~m}, \mathrm{MNRJ} 28741$; 2 specimens, $21^{\circ} 45^{\prime} 2.157^{\prime}$ 'S $40^{\circ} 25^{\prime} 45.051^{\prime \prime} \mathrm{W}, 25$ m, MNRJ 28742; 1 specimen, 22 ${ }^{\circ} 1$ '22.234'"S $40^{\circ} 20^{\prime} 12.811^{\prime \prime} \mathrm{W}, 60 \mathrm{~m}$, MNRJ 28743; 1 female, 2150'20.898''S 40³1'39.721',W, $27 \mathrm{~m}$, MNRJ 28874 (Figure 4). Paraná - 1 specimen, 2536'45.53”'S 48 22'53.85'W, MNRJ 14062; 1 specimen, $25^{\circ} 46.68^{\prime} \mathrm{S} 48^{\circ} 20.64^{\prime} \mathrm{W}, 5 \mathrm{~m}$, MNRJ 21639.

Remarks: Phoxocephalopsi zimmeri was first described based on material from the Swedish Antarctic Expedition for the Magellan province and Falkland Islands (Schellenberg 1931). Barnard and Clark (1984) redescribed P. zimmeri with excellent illustrations based on Schelenberg's 1931 type series. The holotype material seems to be lost, but Barnard and Clark (1984) do not establish a neotype. Subsequently, P. zimmeri was registered along the continental shelf (1-120 m) of the southeast Brazilian coast $\left(23^{\circ}-25^{\circ} \mathrm{S}\right)$ (Wakabara et al. 1991). In the present study, this species prove to be abundant within the Campos Basin region between depths of 16-60 m. Remarkable differences of P. zimmeri and P. ruffoi are discussed above.

Type locality. Magellan Area, Punta Arenas, Chile, $4 \mathrm{~m}$, sand bottom (Schellenberg 1931, De Broyer et al. 2007).

Distribution: Falkland Islands: Port Albemarle (40 m); Berkeley Sound (25-30 m); Discovery 1925-27: sta. WS 96, 4800’S 64²5’W, 96 m; Port Stanley; Magellan Province: Punta Arenas (4 m); Estrecho de Magallanes (4-21 m); southern Tierra del Fuego, Bahia Aguirre (30-35 m); Bahia Buen Suceso (12 m) (Schellenberg 1931, De Broyer et al. 2007). Argentina: $35^{\circ}-56^{\circ} \mathrm{S}$ (Gappa et al.
2006). Brazil: Espírito Santo Basin (32 m); Rio de Janeiro - Campos Basin (16-60 m), Figueira Beach, Arraial do Cabo (30-60 m); Paraná - Atami Beach in Pontal do Sul and Matinhos (5 m) (present work). Santa Catarina (Boos et al. 2012).

\section{Puelche irenae sp. nov.}

Figures 5-8

ZooBank Life Science Identifier (LSID) urn:1sid:zoobank.org:act:5E0BD676-1030-41109F9A-F1F9504C60A4

Holotype: Male, length $5.3 \mathrm{~mm}$, Campos Basin, Rio de Janeiro, $21^{\circ} 45^{\prime} 2.157^{\prime}$ 'S 40 $25^{\circ} 45.051$ 'W, 25 m, MNRJ 28808.

Allotype: Female, length $4.5 \mathrm{~mm}$, Campos Basin, Rio de Janeiro, 2145'2.157'S 40²5'45.051'W, 25 m, MNRJ 28809.

Paratypes: Espírito Santo - 1 male, 1940'25.29”S 39³6'21.96”'W, 34 m, MNRJ 28850; 2 females, 1940'25.29”S - 39³6'21.96”W, 34 m, MNRJ 28851; 1 female 1940'25.29”'S - 39³6'21.96”'W, 34 m, MNRJ 28852; 1 male, 1947'32.83"S 39 43 '15.08'W, 37 m, MNRJ 28853; 1 male, 2 females, 1946'14.99''S - 39³4'50.94'W, 44 m, MNRJ 28854; 1 male, 1 female, 1944'44.06"S - 39०46'32.01”W, 29 m, MNRJ 28855; 1 male, 19³9'47.16”S - 3942'13.45”W, 26 m, MNRJ 28856; 1 male 19०39'47.16"S - 3942'13.45"W, 26 m, MNRJ 28857; 1 male, 1947'22.52”S 3943'20.72"W, 34 m, MNRJ 28858; 1 male, 1 juvenile, 1946'10.69"'S - 39³4'55.84'"W, 44 m, MNRJ 28859; 1 male, 1 juvenile, 1946'10.69's - 39³4'55.84'W, 44 m, MNRJ 28860. Rio de Janeiro - 2 males, 6 females, 22 ${ }^{\circ}$ '22.014' S 4043'42.326"'W, 47 m, MNRJ 28811; 1 female, $21^{\circ} 34$ '12.975's - 40²5'29.009'”W, $28 \mathrm{~m}$, MNRJ 28812; 1 male, 2 females, $21^{\circ} 45^{\prime} 2.157^{\prime \prime} \mathrm{S}$ - 40 $25^{\circ} 45.051^{\prime \prime} \mathrm{W}, 25 \mathrm{~m}$, MNRJ 29813; 1 male, 1 female, 22¹1'30.609' $\mathrm{S}-40^{\circ} 55^{\prime} 42.468^{\prime}$ 'W, 44 m, MNRJ 28815; 3 males, 2143'22.206”'S - 40³1'52.366"'W, 26 m, MNRJ 28816; 1 


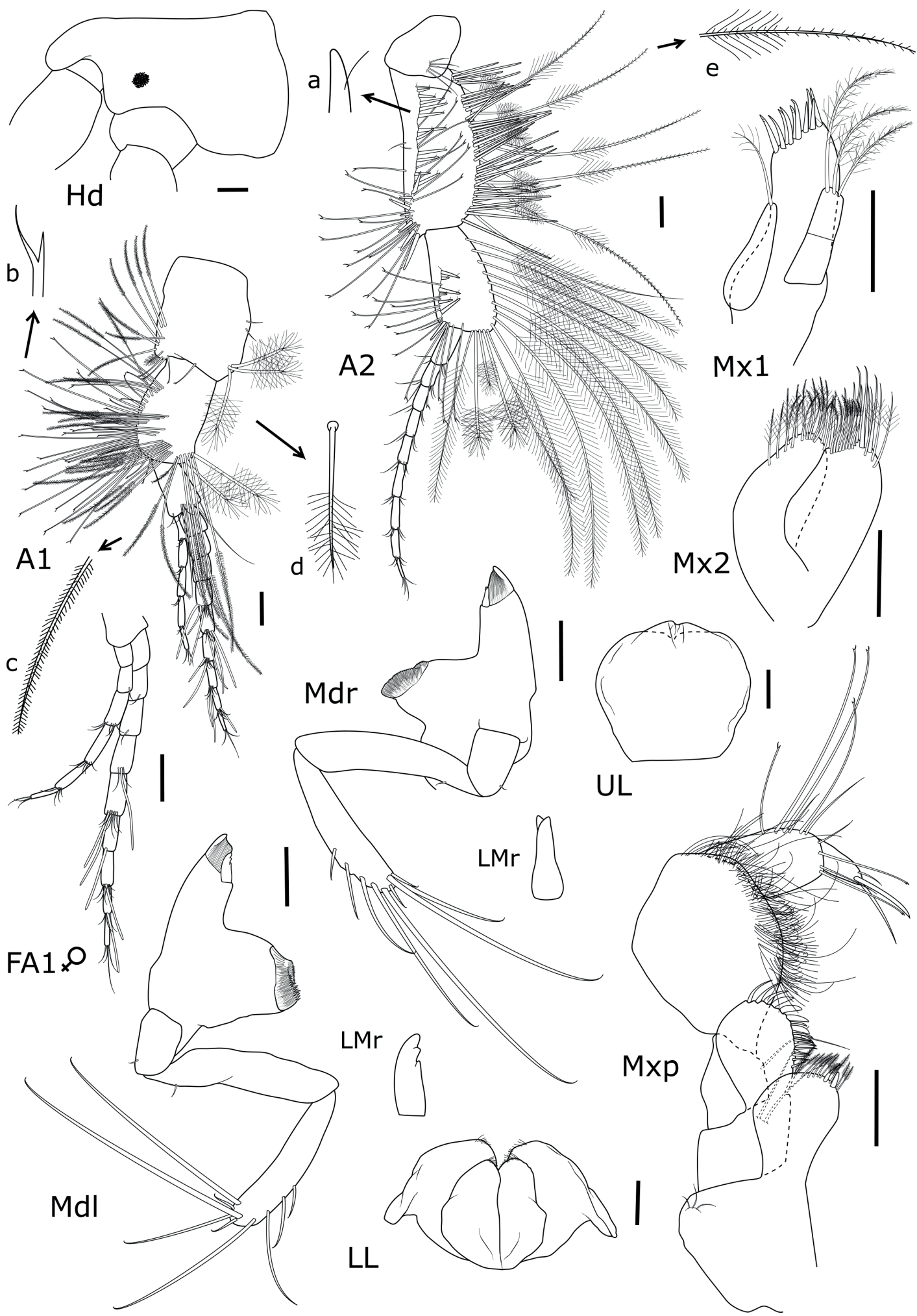

Figure 5 - Puelche irenae sp. nov. Male, 5.3 mm, MNRJ 28808: Head, antennas and mouthparts. Female, 4.5 mm, MNRJ 28809: Flagellum of antenna 1. Detailing of setae of antenna 1: a. robust seta with accessory seta; $\mathbf{b}$. simple seta with lamellate tip; c. plumose seta with small setules; d. penicillate seta. Scales $=0.1 \mathrm{~mm}$. 


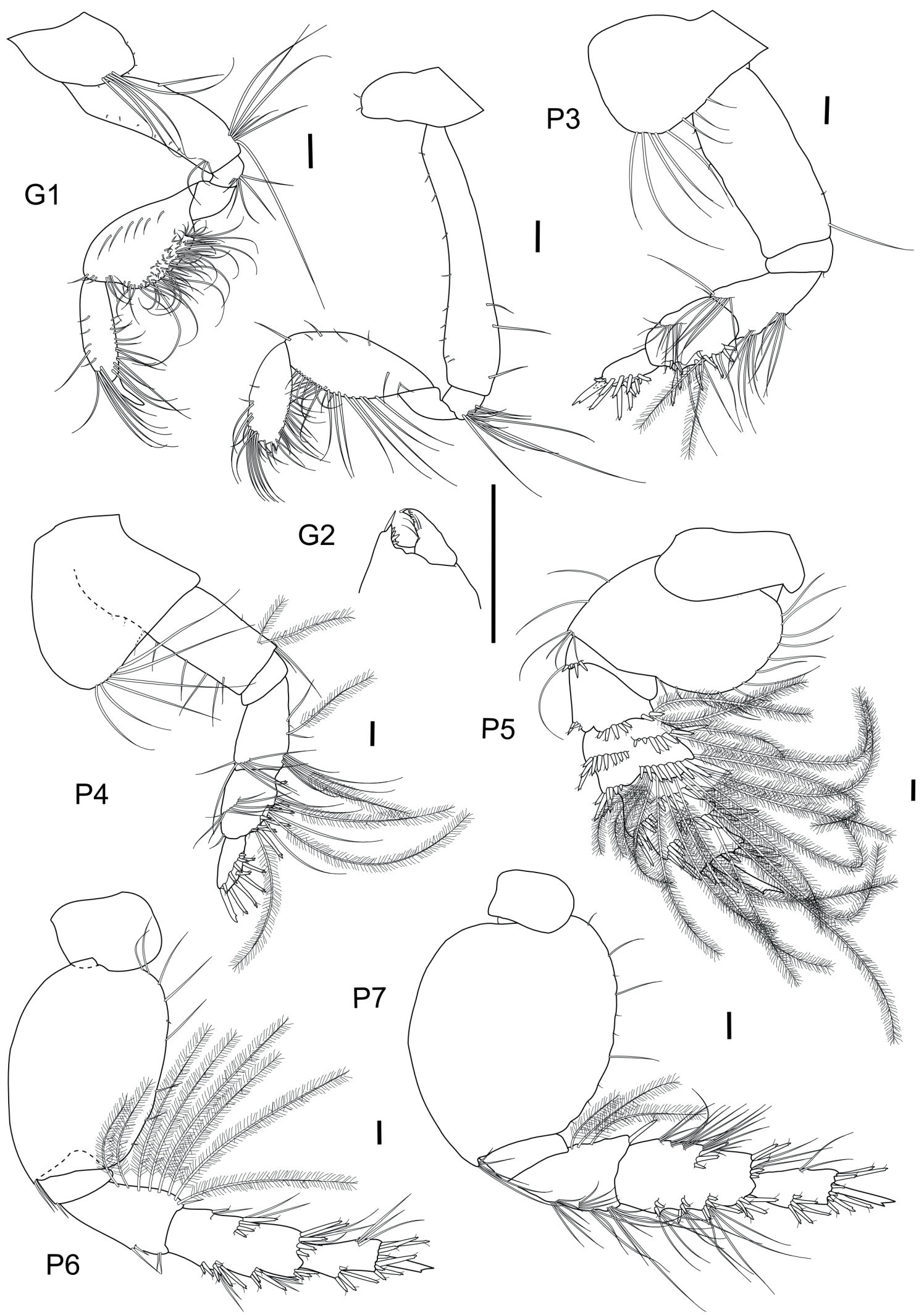

Figure 6 - Puelche irenae sp. nov. Male, $5.3 \mathrm{~mm}$, MNRJ 28808: Gnathopods and pereopods. Scales =0.1 mm. 


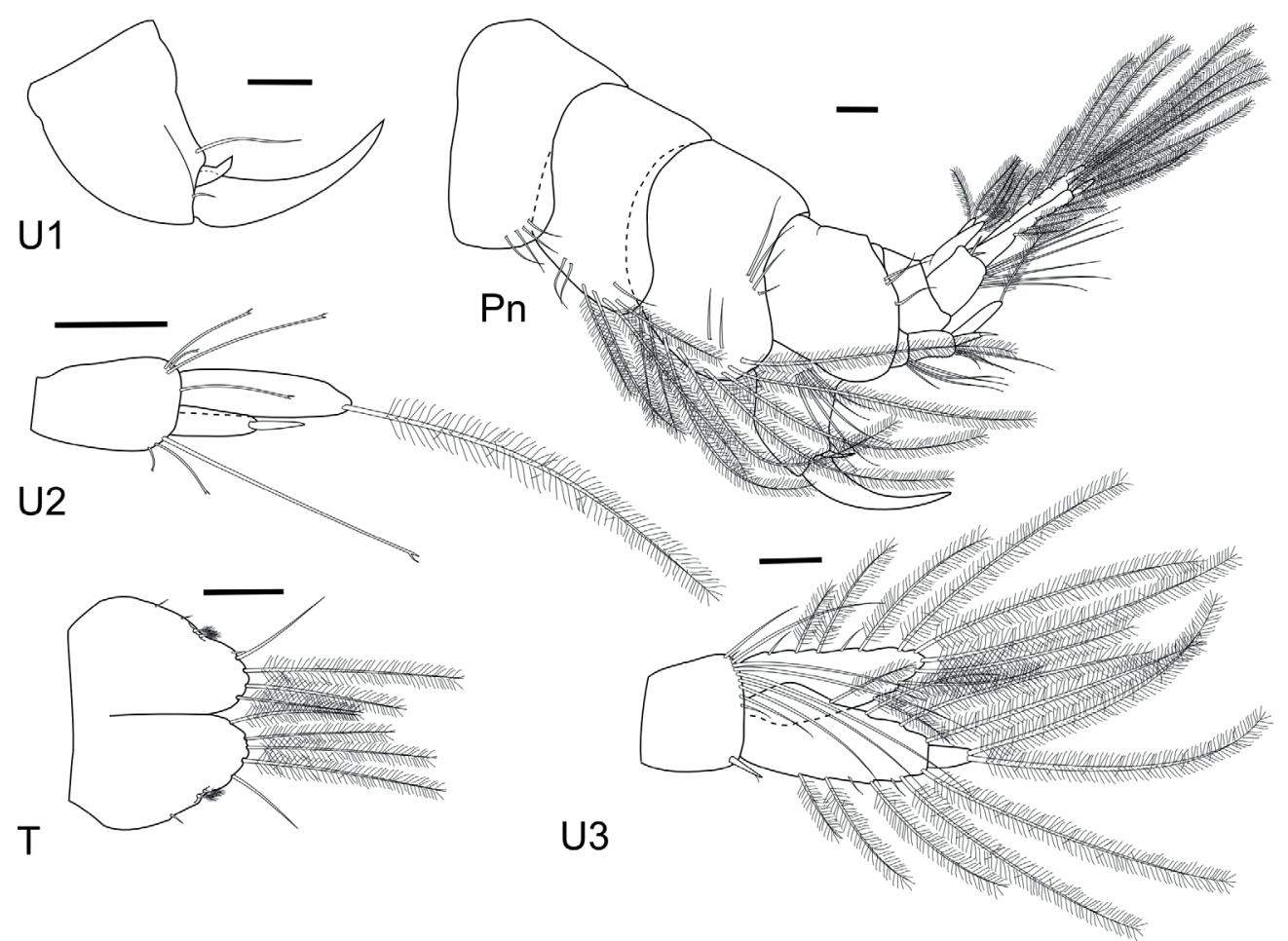

Figure 7 - Puelche irenae sp. nov. Male, $5.3 \mathrm{~mm}$, MNRJ 28808: Uropods, pleon and telson. Scales = $0.1 \mathrm{~mm}$.

male, $21^{\circ} 43^{\prime} 22.278^{\prime \prime} \mathrm{S}-40^{\circ} 31^{\prime} 52.345^{\prime \prime} \mathrm{W}, 26$ $\mathrm{m}$, MNRJ 28817; 1 female, 2150'20.765's 40³1'38.459', 28 m, MNRJ 28818; 2 males, 1 juvenile, $22^{\circ} 6$ '20.060" $\mathrm{S}-40^{\circ} 43^{\prime} 41.632^{\prime}$ W, 47 m, MNRJ 28819; 2 females, 21\%47'12.706's 4057'37.349"'W, 16 m, MNRJ 28820; 2 males, 1 juvenile, $21^{\circ} 55^{\prime} 52.562^{\prime \prime} \mathrm{S}-40^{\circ} 25^{\prime} 56.379^{\prime}$, $47 \mathrm{~m}$, MNRJ 28821; 1 male, 2 females, 1 juvenile, $21^{\circ} 39^{\prime} 9.790$ ' $\mathrm{S}-40^{\circ} 48^{\prime} 50.234^{\prime \prime} \mathrm{W}$, $22 \mathrm{~m}, \mathrm{MNRJ} 28822$; 1 female, $21^{\circ} 23^{\prime} 5.585^{\prime}$ 'W - 40'42'41.149'”W, 28 m, MNRJ 28823; 1 male, $21^{\circ} 17 ' 53.954$ 'S - 4048'45.992"'W, 23 m, MNRJ 28824; 2 males, 1 females, 1

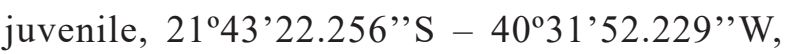
26 m, MNRJ 28825; 1 male, 2139'11.110's 4048'49.729'”, 22 m, MNRJ 28827; 1 male, 1 juvenile, 2128'19.692'"S - 4048'38.086”'W, 22 m, MNRJ 28828; 3 males, 1 female, 226'20,149'"S - 4043'41.039''W, 47 m, MNRJ 28829; 1 male, 1 female, 1 juvenile, 21\%33'54.611's -
4042'53.901' W, 23 m, MNRJ 28830; 2 males, 3 females, 21\%28'19.756's - 4048'34.773, $21 \mathrm{~m}$, MNRJ 28831; 1 male, 2128'2.071'”S 4056'16.956"W, 15 m, MNRJ 28832; 1 female, $21^{\circ} 17^{\prime} 53.022^{\prime \prime S}-40^{\circ} 30^{\prime} 59.297^{\prime \prime} \mathrm{W}, 30 \mathrm{~m}$, MNRJ 28833; 3 males, 3 females, $21^{\circ} 55^{\prime} 50.857^{\prime \prime} \mathrm{S}$ 40²5'59.211' W, $47 \mathrm{~m}$, MNRJ 28834; 1 male, $21^{\circ} 17^{\prime} 54.738^{\prime} \mathrm{S}-40^{\circ} 48^{\prime} 46.520^{\prime \prime} \mathrm{W}, 23 \mathrm{~m}$, MNRJ 28835; 2 female, $22^{\circ} 7^{\prime} 2.275^{\prime} \mathrm{S}-41^{\circ} 2^{\prime} 7.412^{\prime \prime} \mathrm{W}$, 18 m, MNRJ 28836; 3 males, 1 female, 2 juveniles, 21 ${ }^{\circ} 45^{\prime} 1.967$ 'S - 4025'45.211' W, 26 m, MNRJ 28837; 1 male, 1 female, $21^{\circ} 24^{\prime} 43.527^{\prime \prime} \mathrm{S}$ $40^{\circ} 25^{\prime} 20.695^{\prime \prime} \mathrm{W}, 32 \mathrm{~m}$, MNRJ 28838; 1 male, $21^{\circ} 17^{\prime} 52.350^{\prime \prime} \mathrm{S}-40^{\circ} 31^{\prime} 0.169^{\prime} \mathrm{W}, 30 \mathrm{~m}$, MNRJ 28839; 1 male, 215'16.222'"S - 4055'0.928"W, 16 m, MNRJ 28840; 2 females, 21 ${ }^{\circ} 55^{\prime} 50.186$ "S - 40²5'59.434'”W, 47 m, MNRJ 28841; 1 female, $21^{\circ} 17^{\prime} 52.264^{\prime \prime} \mathrm{S}-40^{\circ} 30^{\prime} 59.571^{\prime \prime} \mathrm{W}, 30$ m, MNRJ 28842; 4 males, 2 females, 1 juvenile, $21^{\circ} 28^{\prime} 21.290 " \mathrm{~S}-40^{\circ} 48^{\prime} 34.280$ ' W, $21 \mathrm{~m}$, 


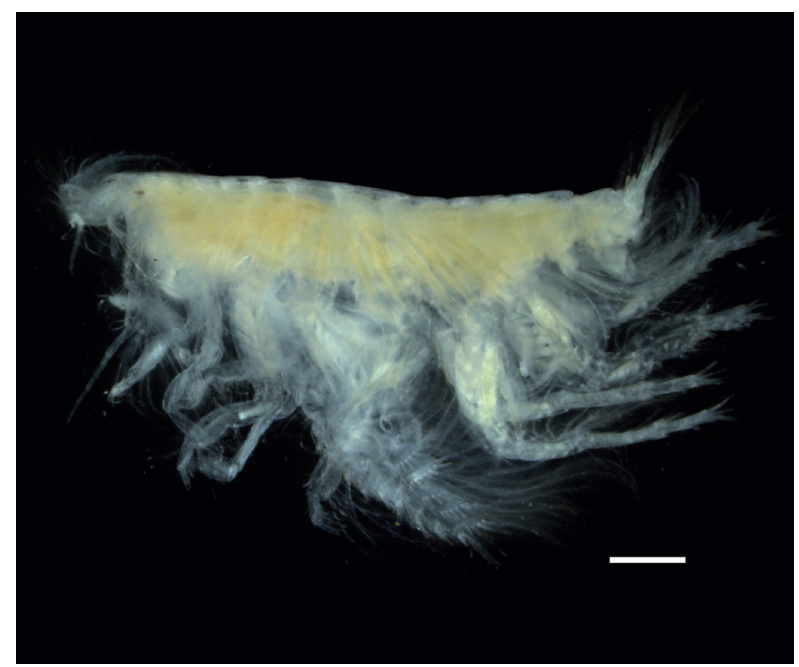

Figure 8 - Puelche irenae sp. nov. Female, MNRJ 28984: Habitus. Scale $=0.5 \mathrm{~mm}$.

MNRJ 28843; 1 male, 1 female, $22^{\circ} 6$ '56.222"S - 40³8'58.224'”, 53 m, MNRJ 28844; 3 males, 1 femnale, $22^{\circ} 6{ }^{\prime} 55.679$ ' $\mathrm{S}-40^{\circ} 38^{\prime} 58.325^{\prime \prime} \mathrm{W}$, 53 m, MNRJ 28845; 1 male, 2159'3.967''S$40^{\circ} 25^{\prime} 10.289^{\prime} \mathrm{W}, 52 \mathrm{~m}$, MNRJ 28846; 1 male, $23^{\circ} 04.266^{\prime} \mathrm{S}-44^{\circ} 21.752^{\prime} \mathrm{W}, 15 \mathrm{~m}$, MNRJ 28847; 2 males, $23^{\circ} 02.601^{\prime} \mathrm{S}-44^{\circ} 8.398^{\prime} \mathrm{W}, 6.5 \mathrm{~m}$, MNRJ 28848; 2 males, 1 female, $23^{\circ} 09.827^{\prime} \mathrm{S}$ $-44^{\circ} 05.941^{\prime} \mathrm{W}, 10 \mathrm{~m}, \mathrm{MNRJ} 28849$; 1 female, 21\%43'22.256"S - 40³1'52.229'”, 26 m, MNRJ 28984 (photographed).

Etymology: Named in honour of Dra. Irene Azevedo Cardoso (Museu Nacional/UFRJ), for her partnership along all these years and significant contribution to studies on Decapod Crustaceans.

Diagnosis: Mandibular incisors not toothed; rakers absent. Coxa $33 x$ larger than coxa 2, subrectangular, reaching about half the length of the basis, ventral angles rounded, without lobes, posterior margin with simple setae; Coxa 4 reaching more than half the length of the basis, with pointed posteroventral lobe, ventral margin with long simple setae. Pereopod 5-7, posterior margin of basis with simple setae, dactylus styliform, shorter than propodus, with distal tooth. Epimeron 3 dominant in size, epimera without sharp points or teeth. Urosomite 1 with posterior lobe rounded, covering urosomite 2 . Uropod 1 biramus, rami styliform, naked, sharply pointed, inner ramus reduced, $0.2 \mathrm{x}$ the length of outer ramus. Uropod 2, rami rodlike, inner ramus longer than outer, with 1 long plumose seta apically; outer ramus with 2 robust setae apically. Telson longer than wide, deeply cleft, lobes tapered, each one with 2 small penicillate setae and 1 long simple seta on outer margin, apex with 3 long plumose setae.

Description: Male, $5.3 \mathrm{~mm}$, MNRJ 28808. Head $1.7 x$ longer than wide, rostrum about $1 / 5$ of the length of head, cheek present, small eyes.

Antenna 1, articles 1-3 of peduncle progressively thinner and shorter; article 1 anterolateral armament with 7 plumose setae with small setules, 1 small penicillate seta and 4 simple setae with lamellate tip, posterodistal angle with 3 penicillate setae; article 2 densely setose, anterior margin with many simple setae with lamellate tip, 6 lateral rows of plumose setae with small setules, posterodistal angle with 2 penicillate setae; article 3 with short simple setae distally; primary flagellum with 9 articles, aesthetascs present, formula $=0-0-0-3-3-2-$ 2-1-0; accessory flagellum with 5 articles. Antenna 2 , article 3 with simple setae laterally; article 4 with anterior row of robust setae with accessory seta and simple setae with lamellate tip, posterior margin covered by robust setae, with 5 penicillate setae and 5 plumose setae (Fig. 9e); article 5 shorter and a little thinner than article $4,0.8 \mathrm{x}$ its width, anterior margin with row of robust setae with accessory seta, anteroventral row of simple setae with lamellate tip, posterior row of plumose setae, and 3 penicillate setae on posteroventral angle; flagellum 9-articulated.

Upper lip rounded, 0.9x longer than wide, naked. Mandibular incisors not toothed; right lacinia with 2 teeth and left lacinia with 3 teeth; rakers absent; molar broad, elongate, triturative in both sides; palp 3-articulated, similar in both sides, article 1 short, $0.3 \mathrm{x}$ the length of the article 
2; article 1-2 with 1 short simple seta each; article $30.9 x$ the length of the article 2 , with 9 distal setae with hooklike tip on each mandible. Lower lip with mandibular lobes well developed, posteriorly directed. Maxilla 1, inner plate tapered, about half the length of outer plate, with 1 plumose seta apically; outer plate with 10 apical robust setae weakly serrate; palp about half the length of outer plate, weakly 2 -articulated, with 3 pappose setae apically. Maxilla 2, plates subequal in length and width; inner plate with apical row of plumose setae and subapical row of plumose and pappose setae; outer plate with apical row of robust setae and subapical row of plumose and pappose setae. Maxilliped, inner plate broad with 1 robust seta and 10 pappose setae apically, 1 of them stubby; outer plate as broad as inner plate, inner margin with 1 proximal pappose seta, many robust setae and simple setae; article 1 of palp with 1 long simple seta on inner margin; article 2 broad, with dense row of setae on inner margin; article 3 thinner than article 2, with simple setae, some with lamellate tip; article 4 rod-shaped with 2 apical robust setae.

Gnathopod 1, coxa ventrally rounded with group of 5 long simple setae; basis and isquium with posterodistal group of simple setae; carpus posteriorly tumescent, densely setose on posterior margin, with sparse row of short simple setae laterally; propodus thinner, with simple setae distally; dactylus simple. Gnathopod 2, coxa ventrally rounded; basis longer than in $\mathrm{G} 1$, with a few short simple setae; setose articles with simple setae most on posterior margin; chelate, palm with 4 robust setae, distally delimited by a acute projection of the propodus and 1 large robust seta, dactylus short with apical flake, inner margin medially lumpy with small simple setae. Pereopods 3 , coxa $3 x$ larger than coxa 2, subrectangular, $1.4 x$ longer than wide, reaching about half the length of the basis, ventral angles rounded, without lobes, posterior margin with simple 8 simple setae; merus with groups of simple setae; carpus posterior margin with 10 robust setae and 2 plumose setae; propodus with robust setae with accessory seta and distal tooth surrounding dactylus (setal formula 6-1-5); dactylus with distal tooth. Pereopod 4, coxa as long as wide, reaching more than half the length of the basis, with pointed posteroventral lobe, ventral margin 6 long simple setae; basis with 2 plumose setae distally; merus with simple setae and plumose setae; carpus, posterior margin with row of simple setae and plumose setae, 10 robust setae with accessory seta and distal tooth; propodus with robust setae surrounding dactylus (setal formula 5-1-5); dactylus with distal tooth. Pereopod 5, coxa bilobed with anterior lobe larger than posterior; basis 1.3 wider than long, with groups of simple setae on anterior margin and 13 simple setae on posterior margin; ischium with group of robust setae on anterior margin; merus, carpus and propodus with groups of robust setae with accessory seta and distal tooth, posterior margins with plumose setae, carpus strongly setose on posteroventral angle; dactylus with distal tooth. Pereopod 6, coxa bilobed with posterior lobe larger than anterior; basis suboval, $1.4 \mathrm{x}$ longer than wide, with 2 simple setae on anteroventral angle and 8 simple setae on posterior margin; merus, posterior margin with plumose setae; merus, carpus and propodus with groups of robust setae with accessory seta and distal tooth; dactylus with distal tooth. Pereopod 7 longer than 6; coxa distally rounded; basis suboval, as long as wide, with group of simple setae on anteroventral angle and 4 simple setae on posterior margin; merus with groups of simple setae on anterior margin, plumose setae on posterior margin and 1 robust seta on posteroventral angle; carpus and propodus with groups of simple setae and groups of robust setae with accessory seta and distal tooth; dactylus with distal tooth.

Epimera distally rouded, lacking posteroventral tooth, with simple setae; epimeron 2-3 with long plumose setae; epimeron 3 dominat. Pleopods in urohaustoriid form. 
Urosomite 1 with posterior lobe rounded, covering urosomite 2, with 3 simple setae on posterior margin. Uropod 1, peduncle with 2 simple setae on dorsal margin; biramus, rami styliform, naked, sharply pointed; inner ramus reduced, $0.2 \mathrm{x}$ the length of outer ramus. Uropod 2, inner and outer apex of peduncle with simple setae with lamellate tip; rami short, rodlike, inner ramus as long as peduncle, with 1 long plumose seta apically; outer ramus about half the length of inner ramus, with 1 apical robust seta with accessory seta. Uropod 3, peduncle as long as wide, dorsal apex with 9 long simple setae, outer apex with 1 robust seta; ramus leaflike, inner ramus 1-articulated, as long as article 1 of outer ramus, inner margin with 4 plumose setae, outer margin with 3 plumose setae, apex with 2 plumose setae; outer ramus 2-articluted, article 1 with 4 plumose setae on inner margin and with 5 plumose setae on outer margin, second article 1/5 of article 1, with 2 plumose setae apically. Telson longer than wide, deeply cleft, lobes tapered, each one with 2 small penicillate setae and 1 long simple seta on outer margin, apex with 3 long plumose setae.

Dimorphism: Female, 4.5 mm, MNRJ 28809. Antenna 1, primary flagellum with the same number of articles as the male, but with less aesthetascs, formula $=0-0-0-1-2-1-1-1-0$. Accessory flagellum with 6 articles. There are no other significant differences between male and female of Puelche irenae.

Remarks: Puelche irenae sp. nov. presents all the diagnostic features of the genus listed by Barnard and Karaman (1991) as incisors untoothed; mandible rakers absent; coxa 2 with less than one third surface area of coxa 3; epimera 1-3 untoothed; epimeron 3 dominant. However, coxae 1 and 2 are not so small when compared to coxae 3 and 4 . Puelche irenae $\mathrm{n}$. sp. has coxa 2 about $1 / 3$ surface area of coxa 3 . The other Puleche species has coxa 2 less than 1/3 surface area of coxa 3 . Even so, the new species has a large size difference between coxae, a diagnostic feature of Pulche. Puelche irenae differs from P. orensanzi Barnard and Clark, 1982 in the following characters: 1) coxa 3 without lobes, posterior margin with 8 simple setae (versus posteroventral lobe present with 4 simple setae); 2) coxa 4 with 6 long simple setae on ventral margin (versus coxa 4 with 13 short simple setae on ventral and posterior margins); 3) pereopods with well developed plumose armament; 4) urosomite 1 wih posterior lobe rounded, covering urosomite 2 (versus posterior lobe pointed not covering urosomite 2); 5) uropod 1, inner ramus reduced, $0.2 \mathrm{x}$ the length of outer ramus (versus half the length of outer ramus); 6) uropod 2, inner ramus $2 x$ longer than outer, with 1 long plumose seta apically (versus rami subequal in length with robust setae apically); 7) telson apex of each lobe with 3 long plumose setae (versus apex of each lobe with 1 robust seta).

Type locality: Campos Basin (21\%45'2.157' S $40^{\circ} 25^{\prime} 45.051$ 'W), $25 \mathrm{~m}$, Rio de Janeiro, Brazil.

Distribution: Espírito Santo - Espírito Santo Basin (26-44 m); Rio de Janeiro - Campos Basin (15-66 m), Ilha da Gipóia, Ilha dos Arrependidos, Ilha Grande, Baía de Ilha Grande (6.5-15 m) (present work).

Puelche longidactylus sp. nov.

Figures 9-12

ZooBank Life Science Identifier (LSID) urn:1sid:zoobank.org:act:CC859355-863F-48F3AEB0-22382AD52E87

Holotype: Female, length $5.0 \mathrm{~mm}$, Campos Basin, Rio de Janeiro, 22 $2^{\circ} 11>34.778 » \mathrm{~S} 40^{\circ} 55^{\prime} 24.191 » \mathrm{~W}$, 44 m, MNRJ 28746.

Allotype: Male, length $2.9 \mathrm{~mm}$, Campos Basin, Rio de Janeiro, 226'20.149'"S 4043'41.039'”W, 47 m, MNRJ 28747.

Paratypes: Bahia - 2 males, 1 female, $12^{\circ} 59.071^{\prime} \mathrm{S}$ $38^{\circ} 32.205^{\prime} \mathrm{W}, 22.5 \mathrm{~m}, \mathrm{MNRJ} 15776$. Espírito Santo - 2 males, 1940'25.29”S 39³6'21,96”W, 34m, 


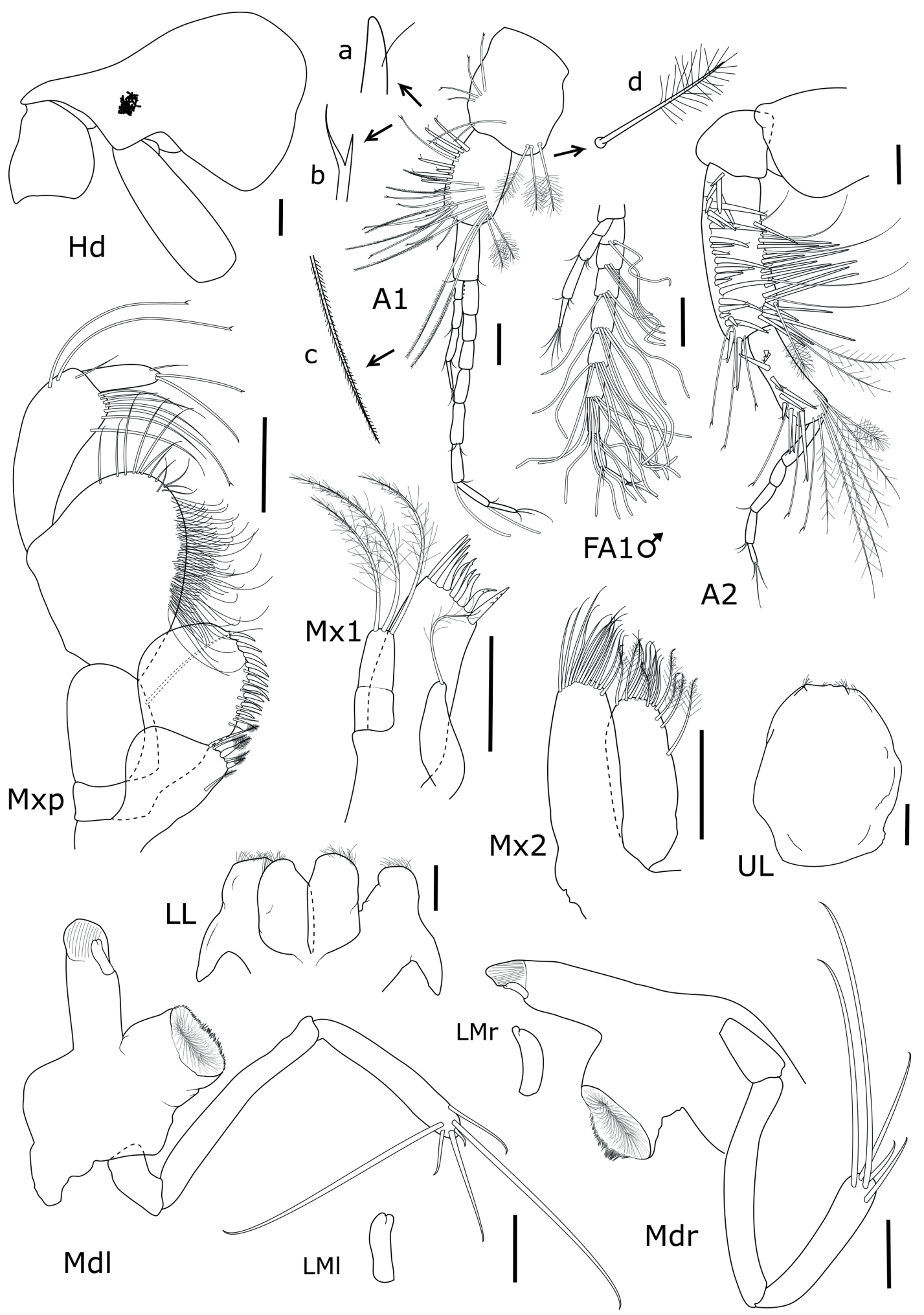

Figure 9 - Puelche longidactylus sp. nov. Female, 5.0 mm, MNRJ 28746: Head, antennae and mouthparts. Male, $2.9 \mathrm{~mm}$, MNRJ 28747: Flagellum of antenna 1. Detailing of setae of antenna 1: a. robust seta with accessory seta; b. simple seta with lamellate tip; c. plumose seta with small setules; d. penicillate seta. Scales $=0.1 \mathrm{~mm}$. 


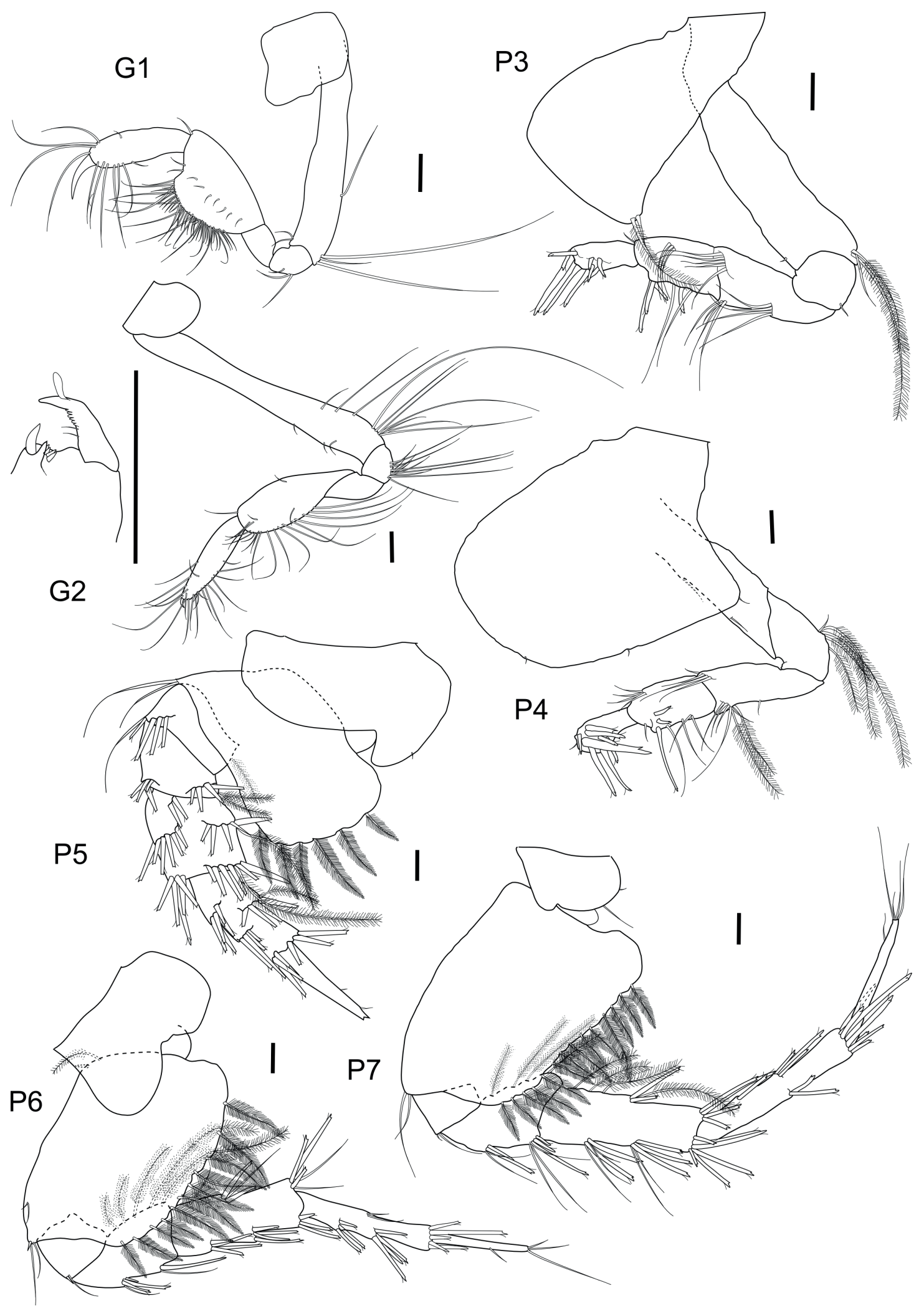

Figure 10 - Puelche longidactylus sp. nov. Female, 5.0 mm, MNRJ 28746: Gnathopods and pereopods. Scales $=0.1$ $\mathrm{mm}$. 

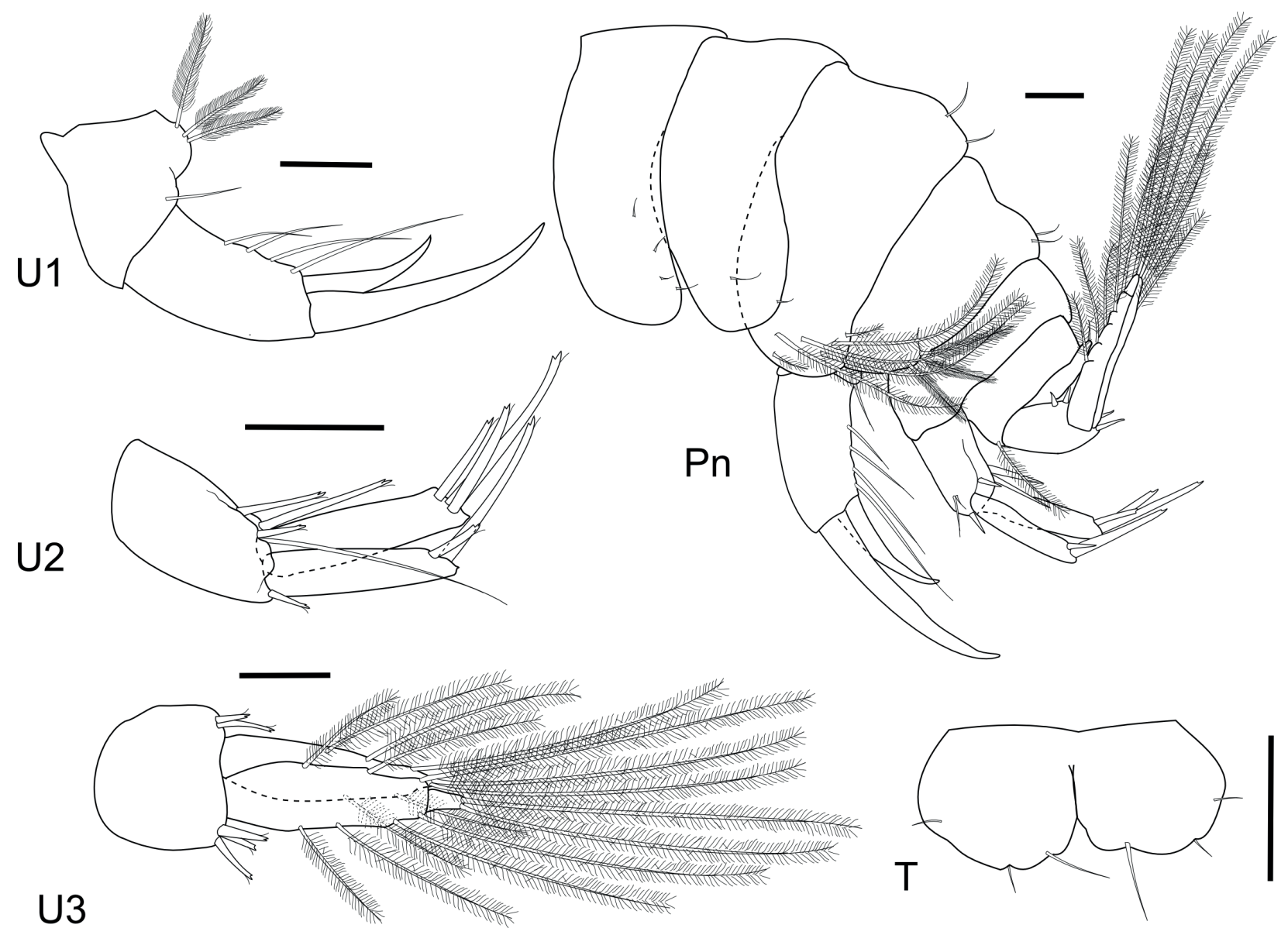

Figure 11 - Puelche longidactylus sp. nov. Female, $5.0 \mathrm{~mm}$, MNRJ 28746: Uropods, pleon and telson. Scales $=0.1 \mathrm{~mm}$.

MNRJ 28785; 1 male, 2 females, 1942’32.21”S 39³8'57.36”'W, 35 m, MNRJ 28786; 1 male, 1 female, $19^{\circ} 52^{\prime} 35.48^{\prime \prime} \mathrm{S}-39^{\circ} 49 \times 5.63 » W, 42$ m, MNRJ 28787; 2 males, 1 female, 1 juvenile, $19^{\circ} 52$ '35.48”S - 3949'5.63”'W, $42 \mathrm{~m}, \mathrm{MNRJ}$ 28788; 2 males, 1 female, $19^{\circ} 52^{\prime} 35.48^{\prime \prime} \mathrm{S}$ 39 49'5.63"W, 42 m, MNRJ 28789; 1 female, $19^{\circ} 54$ '56.16”S 3956'40.83”'W, $32 \mathrm{~m}$, MNRJ 28790; 1 female, $19^{\circ} 47^{\prime} 32.83$ 'S 3943'15.08”W, 37 m, MNRJ 28791; 1 male, 5 females, 1946'14.99'S 39³4'50.94'W, 44 m, MNRJ 28792; 1 male, 1941'24.99”'S 39³1'20.42'"W 44 m, MNRJ 28793; 1 male, $19^{\circ} 47^{\prime} 22.52$ "S 3943'20.72”W, 34 m, MNRJ 28794. Rio de Janeiro - 5 males, 7 females, 1 juvenile, 22 ${ }^{\circ} 11$ '34.778's 4055'24.919'”, 44 m, MNRJ 28748; 1 female, $21^{\circ} 24$ '43.527'"S $40^{\circ} 25$ '20.695”W, 32 m, MNRJ 28749; 1 male,
1 female, $22^{\circ} 6{ }^{\prime} 22.014{ }^{\prime \prime} \mathrm{S}-40^{\circ} 43^{\prime} 42.326^{\prime \prime} \mathrm{W}$, 47 m, MNRJ 28750; 1 male, 21 ${ }^{\circ} 40^{\prime 24.968 ' s}$ 40 58'25.369'”W, $18 \mathrm{~m}$, MNRJ 28751; 1 male, $21^{\circ} 17^{\prime} 52.350$ 'S 40³1'0.169'”W, $30 \mathrm{~m}$, MNRJ 28752; 1 male, 21 '33'54.457' 'S-4042'55.682' 'W, 22 m, MNRJ 28753; 1 female, 21 ${ }^{\circ} 55^{\prime} 25.160$ 'S 4049'12.284"'W, 21 m, MNRJ 28754; 2 males, 21 ${ }^{\circ} 55^{\prime} 30.810 " \mathrm{~S}-40^{\circ} 43^{\prime} 22.824$ 'W, $20 \mathrm{~m}$, MNRJ 28755; 1 male, 2139'31.543' 'S 40³1'25.347'W, 28 m, MNRJ 28756; 1 female, $21^{\circ} 17 ' 53.544$ 's 4048'44.027' 'W, 23 m, MNRJ 28757; 2 females, $21^{\circ} 17 ' 25.438$ 'S - 4054'6.865' W, 16 m, MNRJ 28758; 3 males, 1 female, 1 juvenile, $21^{\circ} 33^{\prime}$ '54.457' S - 4042'55.682'”W, 21 m, MNRJ 28759; 1 male, 3 females, $22^{\circ} 11^{\prime} 30.609$ 'S $40^{\circ} 55^{\prime} 24.468^{\prime \prime} \mathrm{W}$, 44 m, MNRJ 28760; 1 female, 2149'58.813"S 4049'2.818'W, 23 m, MNRJ 28761; 1 female, 


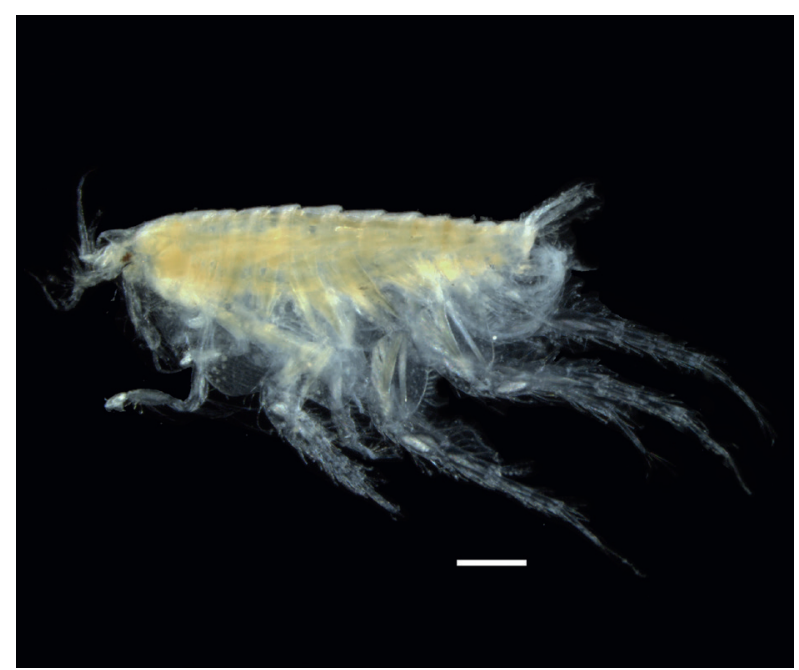

Figure 12 - Puelche longidactylus sp. nov. Female, MNRJ 28985: Habitus. Scale $=0.5 \mathrm{~mm}$.

$21^{\circ} 24^{\prime} 45.072$ 'S - 40²5'17.287'”, 32 m, MNRJ 28762; 1 male, $22^{\circ} 1^{\prime} 22.234^{\prime \prime} \mathrm{S} 40^{\circ} 20^{\prime} 12.811 \mathrm{~W}$, 60 m, MNRJ 28763; 1 male, 2 juveniles, $21^{\circ} 21^{\prime} 21.588^{\prime}$ S 4052'8.331'”, $20 \mathrm{~m}$, MNRJ 28764; 1 female, $21^{\circ} 47^{\prime} 13.287^{\prime \prime} 40^{\circ} 57^{\prime} 37.518^{\prime \prime} \mathrm{W}$, $16 \mathrm{~m}$, MNRJ 28765; 1 male, 1 female, 2 juveniles, 2139'9.790"S 4048'50.234"W, 22 m, MNRJ 28766; 1 male, 2 juvenile, 21 33 '54.340'’S 4042'55.771'’W, $23 \mathrm{~m}$, MNRJ 28767; 1 male, 1 female, $21^{\circ} 24^{\prime} 45.281$ 'S $40^{\circ} 25^{\prime} 19.394$ ' W, $32 \mathrm{~m}$, MNRJ 28768; 1 female, 2128'2.200'"S $40^{\circ} 55^{\prime} 21.516^{\prime \prime} \mathrm{W}, 15 \mathrm{~m}, \mathrm{MNRJ} 28769$; 1 male, 3 females, $21^{\circ} 17^{\prime} 51.743$ 'S $40^{\circ} 30$ '59.011' $\mathrm{W}$, 29 m, MNRJ 28770; 1 female, 22 ${ }^{\circ} 11^{\prime} 32.173$ 's 4055'24.141'”W, 45 m, MNRJ 28771; 2 males, 3 females, $22^{\circ} 6$ '20.149' $\mathrm{S} 40^{\circ} 43^{\prime} 41.039^{\prime}$ 'W, $47 \mathrm{~m}$, MNRJ 28772; 1 male, 1 female, 22 $55^{\circ} 7.577^{\prime} \mathrm{S}$ 400'49.275' 'W, 29 m, MNRJ 28773; 1 male, 1 female, 22 ${ }^{\circ} 55^{\prime} 7.879$ ' $\mathrm{S} 40^{\circ} 0$ ' $49.106^{\prime}$ 'W, $29 \mathrm{~m}$, MNRJ 28774; 8 males, 15 females, 6 juveniles, $22^{\circ} 6$ '42.239'S 4054'44.333'”W, 29 m, MNRJ 28775; 3 females, 7 juveniles, $22^{\circ} 12^{\prime} 53.045^{\prime}$ 'S 4051'12.031W, $52 \mathrm{~m}, \mathrm{MNRJ} 28776$; 1 male, 226'20.060"S 4043'41.632'”W, 47 m, MNRJ 28779; 1 male, 1 females, $21^{\circ} 45^{\prime} 2.327^{\prime \prime} \mathrm{S}$ 40²5'45.451'W, 27 m, MNRJ 28780; 1 males,
3 females, 226'20.149"S 4043'41.039"W, 47 $\mathrm{m}, \mathrm{MNRJ} 28782 ; 8$ male, 1 female, 3 juvenile, $23^{\circ} 04.266^{\prime} \mathrm{S} 44^{\circ} 21.752^{\prime} \mathrm{W}, 15 \mathrm{~m}$, MNRJ 28784; 1 female, $21^{\circ} 45^{\prime} 2.327^{\prime}$ 'S $40^{\circ} 25^{\prime} 45.451^{\prime \prime} \mathrm{W}, 27 \mathrm{~m}$, MNRJ 28985 (photographed).

Etymology: The species name refers to the long and characteristic dactylus with apical setae of pereopods 6 and 7.

Diagnosis: Mandibular incisors not toothed; rakers absent. Coxae 3 and 4 much larger than coxae 1 and 2; coxa 3 subrectangular, reaching the end of the basis, posteroventral lobe present with 1 simple seta and 1 plumose seta. Coxa 4 nearly naked, reaching the end of the ischium, with pointed posteroventral lobe. Pereopods 5-7, posterior margin of basis with many densely setulose plumose setae. Pereopod 5, dactylus as long as propodus, styliform; Pereopods 6-7, propodus and dactylus long, exceeding the distal limit of the body, dactylus rod-shaped with long simple setae apically. Epimeron 3 dominant in size, epimera without sharp points or teeth. Urosomite 1 with posterior lobe pointed and small, not covering urosomite 2. Uropod 1, biramus, rami styliform, naked, sharply pointed, inner about half the length of outer. Uropod 2, rami rodlike, inner ramus slightly longer than outer ramus, with 3 robust setae apically; outer ramus with 2 robust setae apically. Telson $2 x$ wider than long, deeply cleft, lobes rounded, each one with 3 simple setae.

Description: Female, 5.0 mm, MNRJ 28746. Head $1.8 \mathrm{x}$ longer than wide, rostrum about $1 / 5$ of the length of head, cheek present, small eyes.

Antenna 1, articles 1-3 of peduncle progressively thinner and shorter; article 1 anterolateral armament with 6 simple setae with lamellate tip, posterodistal angle with 3 penicillate setae; article 2, anterior margin with simple setae with lamellate tip and robust setae with accessory seta, lateral row of plumose setae with small setules (Fig. 5c), posterodistal angle with 2 penicillate setae; article 3 with 2 short simple setae distally; primary flagellum with 8 articles, aesthetascs 
present, formula $=0-0-0-0-0-1-1-0$; accessory flagellum with 4 articles. Antenna 2, article 3 naked; article 4 with anterior row of robust setae with accessory seta and 3 distal simple setae with lamellate tip, posterior margin with many long robust setae and simple setae, 1 penicillate and 2 plumose setae distally; article 5 shorter and thinner than article 4, 0.6x its width, anterior margin with row of robust setae with accessory seta and 3 distal simple setae with lamellate tip, posterodistal angle with 1 penicillate and 5 plumose setae; flagellum 5-articulated.

Upper lip rounded, 1.2x longer than wide, with small simple setae apically. Mandibular incisors not toothed; right and left lacinia mobilis with 2 blunt teeth; rakers absent; molar broad, elongate, triturative in both sides; palp 3-articulated, similar in both sides, article 1 short, $0.3 \mathrm{x}$ the length of the article 2, both articles naked; article $30.7 x$ the length of the article 2 , with 5 distal setae with hooklike tip on the right mandible and 6 on the left mandible. Lower lip with mandibular lobes well developed, posteriorly directed. Maxilla 1, inner plate tapered, reaching about half the length of outer plate, with 1 plumose seta apically; outer plate with 10 apical robust setae weakly serrate; palp about half the length of outer plate, weakly 2-articulated, with 3 pappose setae apically. Maxilla 2, plates subequal in length and width, both with simple setae apically, inner plate with subapical row of plumose setae. Maxilliped, inner plate broad with 5 pappose setae apically, 3 of them slender and 2 stubby, 1 subapical pappose seta; outer plate broad, larger than inner plate, with robust setae and simple setae on inner margin; article 1 of palp with 1 long simple seta on inner margin; article 2 broad, with dense row of simple setae on inner margin; article 3 thinner than article 2, with simple setae, some with lamellate tip; article 4 rod-shaped with 2 apical robust setae.

Gnathopod 1, coxa small, subquadrate; carpus posteriorly tumescent, densely setose on posterior margin, with sparse row of short simple setae laterally; propodus thinner, with simple setae distally; dactylus simple. Gnathopod 2, coxa small, ventral margin rounded; basis longer than in $\mathrm{G} 1$, with some long simple setae, setose articles with simple setae most on posterior margin; weakly chelate, palm with 4 robust setae, distally delimited by a small acute projection of the propodus and 1 large robust seta, dactylus short with apical flake, inner margin medially lumpy with small simple setae. Pereopod 3, coxa much larger than coxae 1 and 2, subrectangular, $1.8 \mathrm{x}$ longer than wide, reaching the end of the basis, posteroventral lobe present with 1 simple seta and 1 plumose seta; basis with plumose setae on posteroventral angle; merus with groups of simple setae; carpus posterior margin with 4 robust setae; propodus with robust surrounding dactylus(setal formula 4-1-3); dactylus and all robust setae with accessory seta and distal tooth. Pereopod 4, coxa nearly naked, larger than $3,1.3 x$ longer than wide, reaching the end of the ischium, with pointed posteroventral lobe; ischium with a group of plumose setae on posterior margin; merus with simple setae and plumose setae distally; carpus posterior margin with 3 robust setae; propodus with robust setae seta surrounding dactylus, (setal formaula 3-1-3); dactylus and all robust setae with accessory seta and distal tooth. Pereopod 5, coxa bilobed with anterior lobe larger than posterior; basis 1.6x wider than long, with 2 long simple setae on anteroventral angle and posterior margin with 6 plumose setae densely setulose; ischium, merus, carpus and propodus with groups of robust setae with accessory seta and distal tooth, posterior margins with plumose setae; dactylus as long as propodus, styliform, with accessory seta and distal tooth. Pereopod 6, coxa bilobed with lobes subequal; basis subrectangular, 1.3x longer than wide, with 2 simple setae on anteroventral angle and posterior margin with 11 plumose setae densely setulose; merus, posterior margin with plumose setae; merus, carpus and propodus with groups of 
robust setae with accessory seta and distal tooth; dactylus long, rod-shaped with 3 long simple setae apically. Pereopod 7 longer than 6; coxa bilobed with posterior lobe larger than anterior; basis subrectangular, 1.5x longer than wide, with 2 simple setae on anteroventral angle and posterior margin with 14 plumose setae densely setulose; merus and carpus with plumose setae on posterior margin; merus carpus and propodus with groups of robust setae with accessory seta and distal tooth; dactylus long, rod-shaped with 4 long simple setae apically.

Epimeron distally rouded, lacking posteroventral tooth; epimeron 1-2 with a few simple setae; epimeron 3 dominat with long and short plumose setae. Pleopods in urohaustoriid form.

Urosomite 1 with posterior lobe pointed and small, not covering other urosomites, with 3 plumose setae densely setulose; urosomite 3 with 1 long plumose seta on posteroventral angle. Uropod 1, peduncle with 1 row of simple setae dorsally; biramus, rami styliform, naked, sharply pointed, inner ramus about half the length of outer ramus. Uropod 2, dorsal margin of peduncle with 1 long simple seta and 3 robust setae with accessory seta and distal tooth, outer ventral apex with 1 robust seta; rami rodlike, inner ramus slightly longer than outer ramus, apex of rami with robust setae with accessory seta and distal tooth, 3 on inner ramus and 2 on outer ramus. Uropod 3, peduncle wider than long, with 2 robust setae with accessory seta and distal tooth on inner apex, and 3 on outer apex; ramus leaflike, inner ramus 1 -articulated, as long as article 1 of outer ramus, inner margin with 3 plumose setae, outer margin with 2 plumose setae, apex with 2 plumose setae; outer ramus 2 -articluted, article 1 with 3 plumose setae on inner margin and 4 plumose setae on outer margin, second article 1/6 of article 1, with 2 plumose setae apically. Telson $2 \mathrm{x}$ wider than long, deeply cleft, lobes rounded, each one with 3 simple setae.
Dimorphism: Male, $2.9 \mathrm{~mm}$, MNRJ 28747. Antenna 1, primary flagellum with 9 articles, with more aesthetascs than female, formula $=2-3-4-5-6$ 7-2-1-0. There are no other significant differences between male and female of Puelche longidactylus sp. nov.

Remarks: Puelche longidactylus sp. nov. presents all the diagnostic features of genus Puelche listed by Barnard and Karaman (1991) (see remarks from P. irenae). Puelche longidactylus differs from $P$. orensanzi Barnard and Clark, 1982 in the following characters: 1) coxa 3 posteroventral lobe with 1 simple seta and 1 plumose seta (versus 4 simple setae); 2) coxa 4 nearly naked (versus setose with well developed setae); 3 ) pereopods 5-7, posterior margin of basis with many plumose setae densely setulose (versus posterior margin with a few short simple setae); 4) pereopods 6 and 7 dactylus long, rod-shaped with long simple setae apically (versus styliform dactylus with one distal tooth); 5) telson with 3 simple setae on each lobe (versus robust and penicillate setae).

Type locality: Campos Basin $\left(22^{\circ} 11>34.778 » \mathrm{~S}\right.$ 4055'24.191» W), 44 m, Rio de Janeiro, Brazil.

Distribution: Bahia - Todos os Santos Bay (22.5 m); Espírito Santo - Espírito Santo Basin (10 - 44 m); Rio de Janeiro - Campos Basin (15 - 56 m), Ilha da Gipóia, Baía de Ilha Grande (15 m).

Puelche mourae sp. nov.

Figures 13-16

ZooBank Life Science Identifier (LSID) urn:1sid:zoobank.org:act:99B50302-1D4C-41099722-49A676FC978D

Holotype: Female, length $4.0 \mathrm{~mm}$, Campos Basin, Rio de Janeiro, 22¹1'34.778'S 4055'24.191” W, 44 m, MNRJ 28795.

Allotype: Male, length $2.7 \mathrm{~mm}$, Campos Basin,

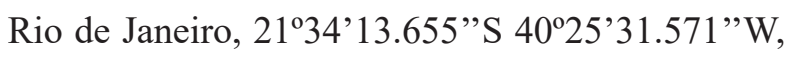
28 m, MNRJ 28796. 
Paratypes: Rio de Janeiro - 1 female, $22^{\circ} 122^{\prime} 53.045^{\prime \prime} \mathrm{S} 40^{\circ} 51 ' 12.0311^{\prime \prime} \mathrm{W}, 52 \mathrm{~m}$, MNRJ 28797; 1 male, 2 females, 1 juvenile, $22^{\circ} 11$ '30.609"S 4055'24.468' W, 44 m, MNRJ

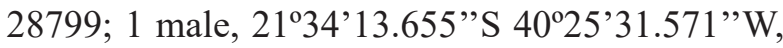
28 m, MNRJ 28800; 1 juvenile, 22 6'20.149'’S 4043'41.039' 'W, 47 m, MNRJ 28801; 3 males, $22^{\circ} 11^{\prime} 31.926^{\prime \prime S} 40^{\circ} 55^{\prime} 24.422^{\prime \prime} \mathrm{W}, 44 \mathrm{~m}$, MNRJ 28802; 1 male, 1 juvenile, $22^{\circ} 11^{\prime} 43.778$ ' $\mathrm{S}$ 4055'24.919W, 44 m, MNRJ 28803; 1 female, 213'12.975"'S 40²5'29.009' W, 28 m, MNRJ 28804; 1 females, $22^{\circ} 6$ '20.060"'S 4043'41.632'”W, 47 m, MNRJ 28805; 2 females, 2134'12.572's $40^{\circ} 25^{\prime} 32.411$ 'W, $29 \mathrm{~m}, \mathrm{MNRJ} 28806$; 1 male, $22^{\circ} 6^{\prime} 22.014$ ' $\mathrm{S} 40^{\circ} 43^{\prime} 42.326^{\prime \prime} \mathrm{W}, 47 \mathrm{~m}$, MNRJ 28807; 1 females, 22 6'20.060'S 4043'41.632'”W, 47 m, MNRJ 28986 (photographed).

Etymology: Named in honor of Mônica Glória Pereira de Moura for her dedication for nearly 10 years as a curator technician of the Crustacea Collection of Museu Nacional/UFRJ.

Diagnosis: Mandibular incisors not toothed; rakers absent. Coxae 3 and 4 much larger than coxae 1 and 2; coxa 3 subrectangular, reaching about half the length of the basis, with anteroventral lobe, with 1 long simple seta on posteroventral angle. Coxa 4 nearly naked, reaching less than half the length of the basis, with rounded anteroventral lobe. Pereopod 5, posterior margin of basis with long plumose setae. Pereopod 6, posterior margin of basis with long simple setae. Pereopods 7, posterior margin of basis with plumose setae densely setulose. Pereopods 5-7, dactylus styliform, shorter than propodus with distal tooth. Epimeron 3 dominant in size, epimera without sharp points or teeth. Urosomite 1 with posterior lobe pointed and small, not covering urosomite 2. Uropod 1, uniramus, ramus styliform, naked, sharply pointed. Uropod 2, rami short, rodlike, inner ramus shorter than outer ramus; inner ramus with 1 robust seta apically; outer ramus with 2 robust apically. Telson longer than wide, deeply cleft, lobes tapered, each one with 1 long simple seta with lamellate tip on the apex.

Description: Female, $4.0 \mathrm{~mm}$, MNRJ 28795. Head $1.5 \mathrm{x}$ longer than wide, rostrum about $1 / 6$ of the length of head, cheek present, medium eyes with sparse ommatidia.

Antenna 1, articles 1-3 of peduncle progressively thinner and shorter; article 1 anterolateral armament with 5 simple setae with lamellate tip and 2 penicillate setae, posterodistal angle with 3 penicillate setae; article 2, anterior margin with simple setae with lamellate tip and robust setae with accessory seta, 2 lateral rows of plumose setae with small setules, posterodistal angle with 1 penicillate seta; article 3 with 1 short simple seta distally; primary flagellum with 9 articles, aesthetascs present, formula $=0-0-0-0-0-2$ 2-2-0; accessory flagellum with 3 articles. Antenna 2 , article 3 with 1 simple seta; article 4 anterior margin with row of small simple setae, robust setae with accessory seta and simple setae with lamellate tip, posterior margin with some long robust setae and simple setae, distal half with 3 penicillate setae and 1 plumose seta; article 5 shorter and thinner than article 4, 0.6x its width, anterior margin with row of robust setae with accessory seta and 1 distal simple seta with lamellate tip, posterior margin with 3 penicillate and 5 long simple setae distally; flagellum 6-articulated.

Upper lip rounded, as long as wide, with small simple setae apically. Mandibular incisor not toothed; right lacinia pointed, 1-toothed, left lacinia mobilis with 3 teeth; rakers absent; molar broad, elongate, strongly triturative in both sides; palp 3 -articulated, similar in both sides, article 1 short, $0.2 \mathrm{x}$ the length of the article 2 , both articles naked; article $30.8 x$ the length of the article 2 , with 5 distal setae with hooklike tip on the right mandible and 6 on the left. Lower lip with mandibular lobes well developed, posteriorly directed. Maxilla 1, inner plate tapered, reaching about half the length of outer plate, with 1 plumose seta apically; outer 


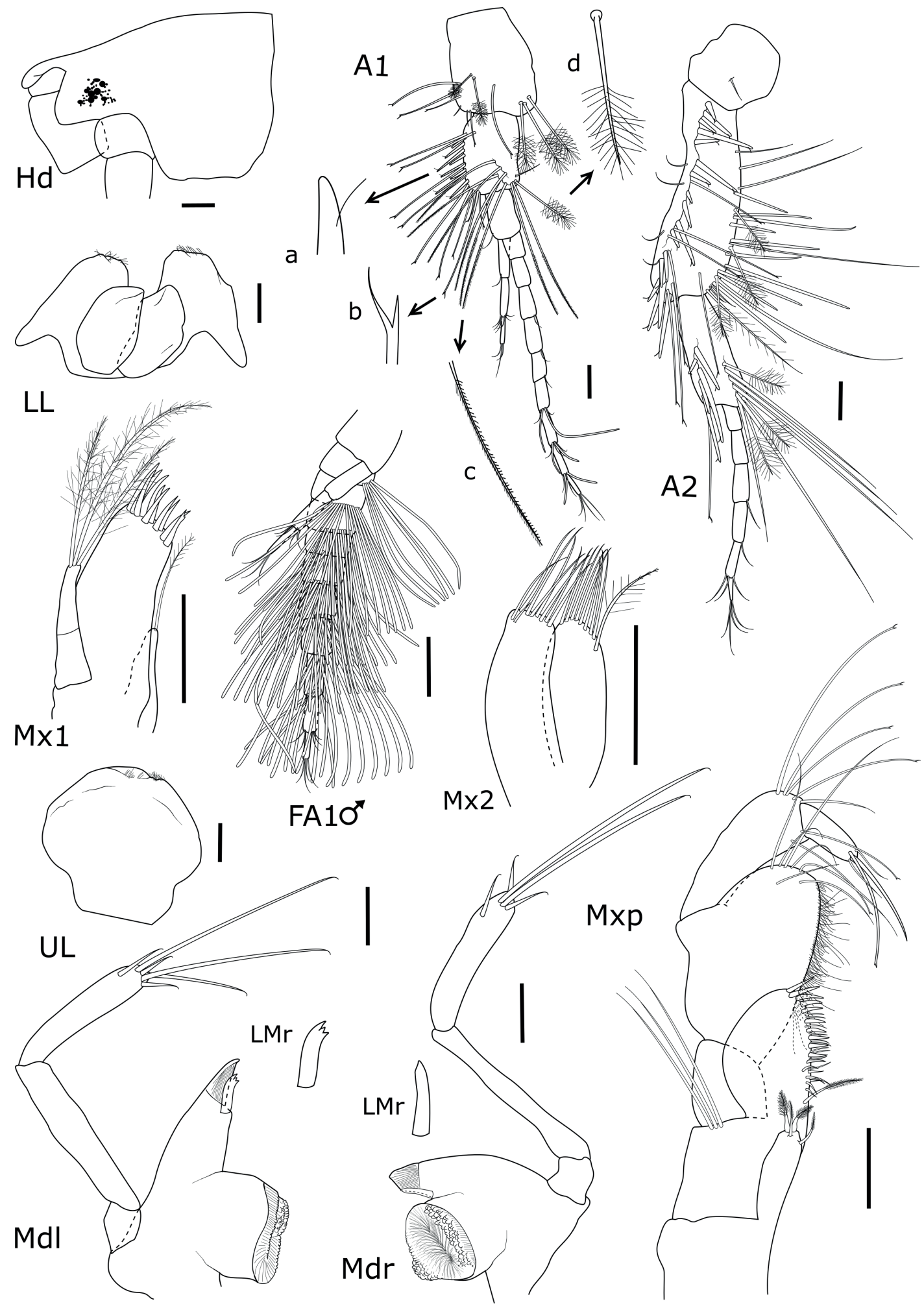

Figure 13 - Puelche mourae sp. nov. Female, 4.0 mm, MNRJ 28795: Head, antennae and mouthparts. Male, 2.7 mm, MNRJ 28796: Flagellum of antenna 1. Detailing of setae of antenna 1: a. robust seta with accessory seta; $\mathbf{b}$. simple seta with lamellate tip; c. plumose seta with small setules; d. penicillate seta. Scales $=0.1 \mathrm{~mm}$. 


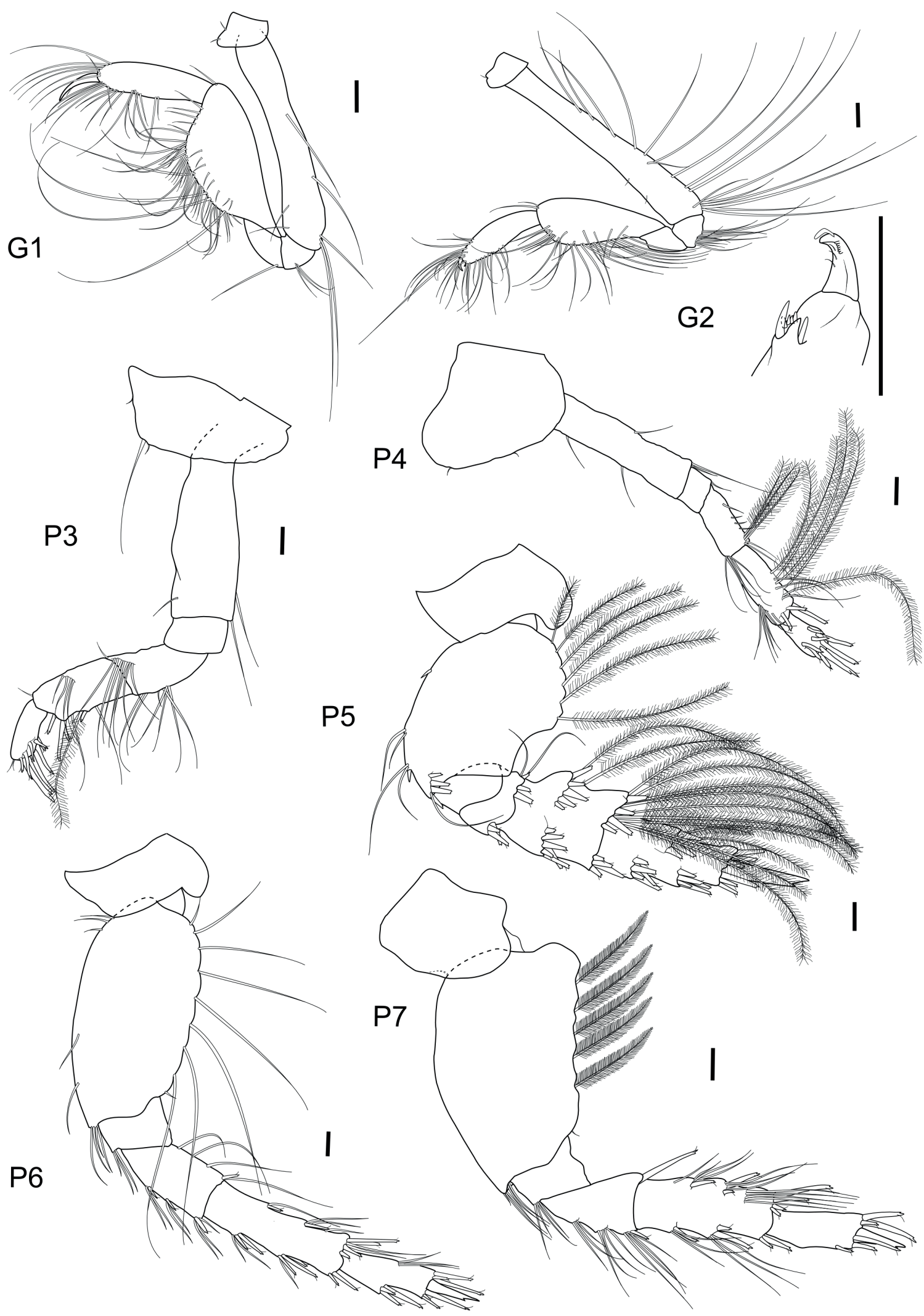

Figure 14 - Puelche mourae sp. nov. Female, 4.0 mm, MNRJ 28795: gnathopods and pereopods. Scales $=0.1 \mathrm{~mm}$. 


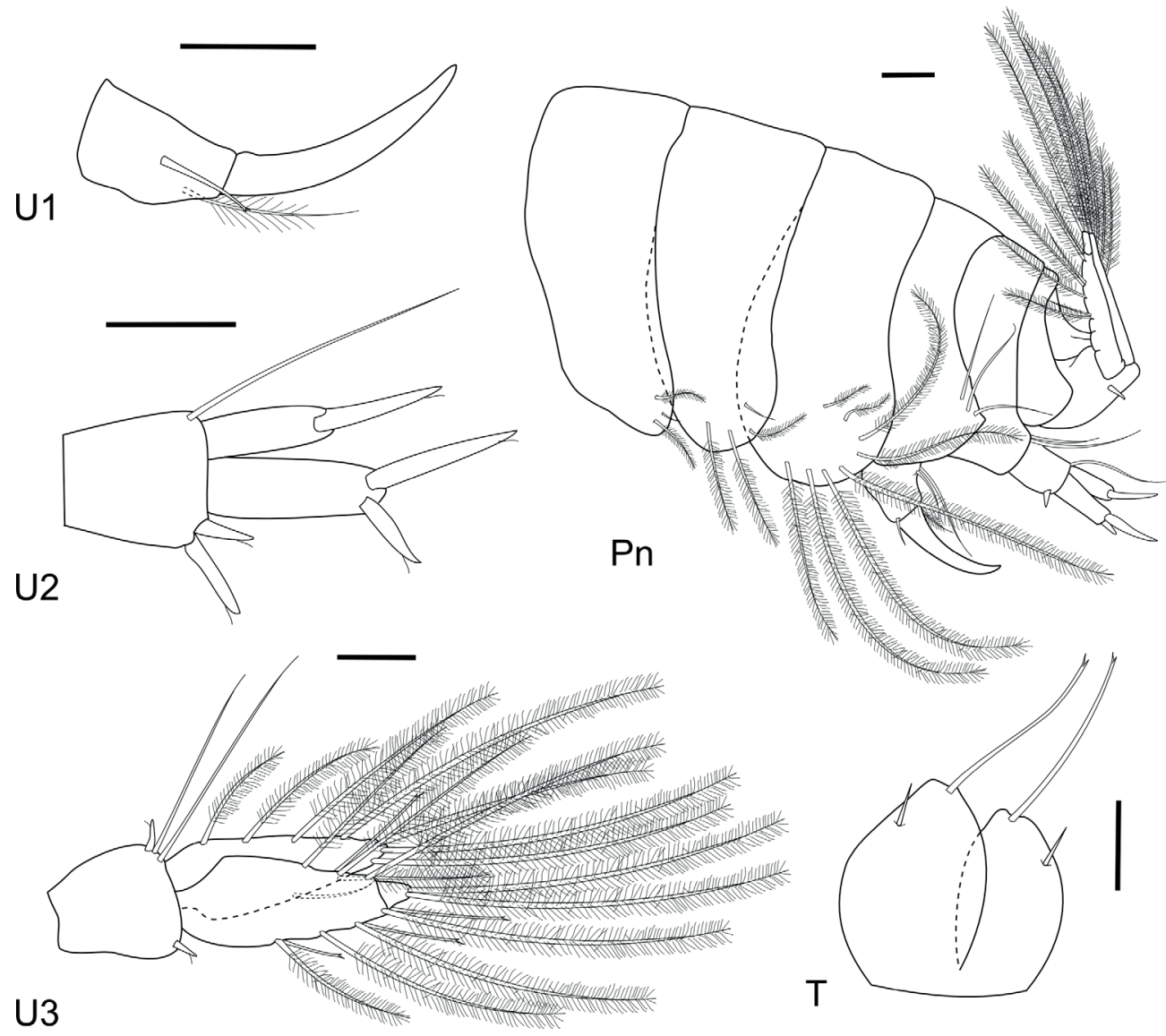

Figure 15 - Puelche mourae sp. nov. Female, 4.0 mm, MNRJ 28795: Uropods, pleon and telson. Scales $=0.1 \mathrm{~mm}$.

plate with 10 apical robust setae weakly serrate; palp about half the length of outer plate, weakly 2-articulated, with 3 pappose setae apically. Maxilla 2, plates subequal in length and width, both with simple setae apically, inner plate with 1 large plumose seta on inner margin. Maxilliped, inner plate thin with 4 pappose setae apically; outer plate broad, inner margin with 1 proximal pappose seta, many robust setae and simple setae, 3 long simple setae facially, close outer margin; article 1 of palp naked; article 2 broad, with dense row of setae on inner margin; article 3 thinner than article 2 , with simple setae with lamellate tip; article 4 of the palp rod-shaped, apex with 1 robust seta and 1 simple seta with lamellate tip.

Gnathopod 1, coxa very small, subquadrate; carpus with posterior tumescence, densely setose on posterior margin, with sparse row of short simple setae laterally; propodus thinner, with simple setae distally; dactylus simple. Gnathopod 2, coxa very small, ventral margin rounded; basis longer than in G1, with many long simple setae posterior margin; setose articles with simple setae most on posterior margin; weakly chelate, palm with 4 robust setae, distally delimited by a small acute projection of the propodus and 2 large robust setae, dactylus short with apical flake, inner margin medially lumpy with small simple setae. Pereopod 3, coxa much larger than coxae 1 and 2 , subrectangular, $1.8 \mathrm{x}$ longer than wide, reaching about half the length of the basis, with anteroventral lobe, with 1 long simple seta on posteroventral angle; merus with groups of simple setae; carpus, posterior margin with 4 robust setae with accessory seta and distal tooth and 1 plumose 


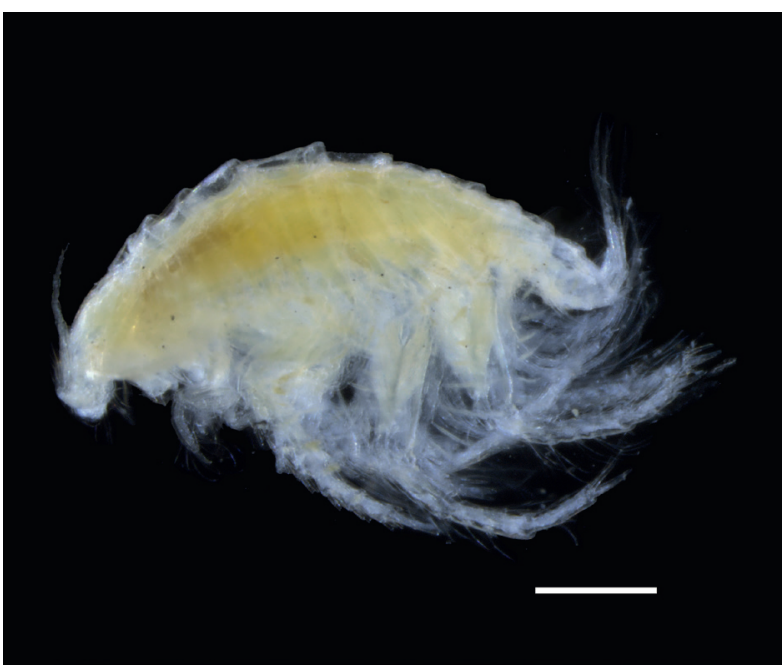

Figure 16 - Puelche mourae sp. nov. Female, MNRJ 28986: Habitus. Scale $=0.5 \mathrm{~mm}$.

seta; propodus with robust setae with accessory seta and distal tooth surrounding dactylus (setal formula 3-1-3); dactylus with distal tooth. Pereopod 4, coxa nearly naked, as long as wide, reaching less than half the length of the basis with rounded anteroventral lobe; merus with simple setae and plumose setae distally; carpus posterior margin with 3 robust setae and row of plumose setae; propodus with robust setae surrounding dactylus (setal formula 3-1-3); dactylus and all robust setae with accessory seta and distal tooth. Pereopod 5, coxa bilobed with anterior lobe larger than posterior; basis as long as wide, with groups of simple setae and robust setae on anterior margin and 6 long plumose setae on posterior margin; ischium short and naked; merus, posterior margin with simple setae; carpus and propodus with plumose setae on posterior margins, most of them on posteroventral angle of carpus; merus, carpus and propodus with groups of robust setae with accessory seta and distal tooth; dactylus with distal tooth. Pereopod 6, coxa bilobed with anterior lobe larger than posterior; basis suboval, $2 \mathrm{x}$ longer than wide, with 8 simple long setae on posterior margin; merus, posterior margin with simple long setae; merus, carpus and propodus with groups of robust setae with accessory seta and distal tooth; dactylus with distal tooth. Pereopod 7 longer than 6; coxa distally rounded; basis $1.7 x$ longer than wide, with group of simple setae on anteroventral angle and posterior margin with 5 plumose setae densely setulose; merus with groups of simple setae on anterior margin, anteroventral and posteroventral angles each one with 1 robust seta with accessory seta and distal tooth; carpus with groups of simple setae and groups robust setae with accessory seta and distal tooth; propodus with distal group of robust setae with accessory seta and distal tooth; dactylus with distal tooth.

Epimeron distally rouded, lacking posteroventral tooth; epimeron 1-3 with plumose setae; epimeron 3 dominat. Pleopods in urohaustoriid form.

Urosomite 1 with posterior lob pointed and small, not covering other urosomites, with 3 simple setae posterior margin. Uropod 1, peduncle with 1 plumose seta on inner margin and 1 simple seta with lamellate tip on outer margin; uniramus, ramus styliform, naked, sharply pointed. Uropod 2 , peduncle with 1 simple seta on inner apex and 2 robust setae with accessory seta on outer apex; rami short, rodlike, inner ramus $0.8 \mathrm{x}$ the length of peduncle, with 1 apical robust seta with accessory seta; outer ramus $1.2 x$ the length of peduncle, longer than inner ramus, with 2 apical robust setae with accessory seta. Uropod 3, peduncle as long as wide, inner apex with 2 long simple setae, inner and outer apex each on with 1 robust seta with accessory seta; ramus leaflike, inner ramus 1 -articulated, as long as article 1 of outer ramus, inner margin with 4 plumose setae, outer margin with 3 plumose setae, apex with 2 plumose setae; outer ramus 2-articluted, article 1 inner margin with 3 plumose setae and 2 simple setae with lamellate tip, outer margin with 4 plumose setae and 3 simple setae with lamellate tip, second article 1/6 of article 1 , with 2 plumose setae apically. Telson longer than wide, deeply cleft, lobes tapered, each one with 1 
robust seta on outer margin and 1 long simple seta with lamellate tip on the apex.

Dimorphism: Male, $2.7 \mathrm{~mm}$, MNRJ 28796. Antenna 1, primary flagellum with 10 articles and many aesthetascs from article 1 to article 8 . There are no other significant differences between male and female of Puelche mourae.

Remarks: Puelche mourae sp. nov. presents all the diagnostic features of genus Puelche listed by Barnard and Karaman (1991) (see remarks from P. longidactylus). P. mourae differs from Puelche orensanzi Barnard and Clark, 1982 in the following characters: 1) coxae 3 and 4 with anteroventral lobe instead of posteroventral lobe; 2) coxa 3 posteroventral angle with 1 long simple seta (versus 4 simple setae); 3 ) coxa 4 nearly naked (versus setose); 4) pereopod 5, posterior margin of basis with 6 long plumose setae (versus 9 short simple setae); 5) pereopod 6 , posterior margin of basis with 8 long simple setae (versus a few short simple setae); 6) pereopods 7 , posterior margin of basis with plumose setae densely setulose (versus posterior margin nearly naked); 7) uropod 1 uniramus (versus biramus); 8) telson with 1 long simple seta with lamellate tip on the apex of each lobe (versus short robust setae).

Type locality: Campos Basin (22 $11^{\prime} 34.778^{\prime \prime S}$ 4055'24.191” W), 44 m, Rio de Janeiro, Brazil.

Distribution: Rio de Janeiro - Campos Basin (28-52 m).

\section{Key to Phoxocephalopsidae from Brazil including Puelche orensanzi}

1) Epimeron 2 dominant; epimera with posteroventral points, mandible with rakers. Phoxocephalopsis (2)

Epimeron 3 dominant; epimera without posteroventral points, mandible lacking rakers Puelche (3)

2) Antenna 2, article 2 with ensiform process truncate apically (Fig. 4); dactylus of pereopod 5 with four groups of robust setae (Fig. 3). P. ruffoi sp. nov.
Antenna 2, article 2 with ensiform process acute apically (Fig. 4); dactylus of pereopod 5 lacking robust setae ................ P. zimmeri Schellenberg, 1931

3) Coxas 3-4 with anteroventral lobe; uropod 1 uniramus (Figs. 14-15)...... P. mourae sp. nov. Coxas 3-4 with posteroventral lobe; uropod 1 biramus ........................................ 4

4) Pereopods 5-7, posterior margin of basis with many plumose setae densely setulose; pereopod 5, dactylus as long as propodus; pereopods 6-7, propodus and dactylus long, exceeding the distal limit of the body, dactylus rod-shaped with long simple setae apically (Fig. 10) P. longidactylus $\mathrm{sp}$. nov.

Pereopods 5-7, posterior margin of basis with simple setae; pereopod 5, dactylus about half the length of propodus; pereopods 6-7 not exceeding the distal limit of the body, dactylus styliform with distal tooth 5

5) Urosomite 1 with posterovetral lobe rounded, covering urosomite 2; uropod 1 inner ramus reduced, $0.2 x$ the length of outer ramus; uropod 2 , inner ramus $2 x$ longer than outer, with 1 long plumose seta apically; telson apex with long plumose setae (Fig. 7) ...... P. irenae sp. nov. Urosomite 1 with posterovetral lobe pointed, not covering urosomite 2; uropod 1 inner ramus half the length of outer ramus; uropod 2 , rami subequal in length with robust setae apically; telson apex of each lobe with 1 robust seta ......... P. orensanzi Barnard and Clark, 1982

\section{ACKNOWLEDGMENTS}

The authors are grateful to Centro de Pesquisas Petróleo Brasileiro S/A (CENPES/PETROBRAS) for providing most of the material. This study is also part of the Rapid Assessment of Biodiversity Ilha Grande Bay Program, which was supported by Projeto de Conservação e Utilização Sustentável 
da Diversidade Biológica Brasileira - PROBIO; Conselho Nacional de Desenvolvimento Científico e Tecnológico (CNPq); Universidade do Estado do Rio de Janeiro - UERJ; Museu Nacional/ Universidade Federal do Rio de Janeiro - UFRJ; Instituto de Pesquisas Jardim Botânico do Rio de Janeiro e Programa das Nações Unidas para o Desenvolvimento - PNUD - Projeto BRA/00-021. We also want to thank Frederico Monteiro Neves for collecting and sending specimens from Rio Grande do Sul. The first author thanks Fundação de Amparo à Pesquisa do Estado do Rio de Janeiro (FAPERJ) for an undergraduate grant process no. E-26/102.340/2011. The second author also thanks CNPQ for productivity research grant process no. 312343/2015-9.

\section{REFERENCES}

BARNARD JL AND CLARK J. 1982. Puelche orensanzi, new genus, new species, a phoxocephalopsid amphipod from the shores of Argentina (Crustacea, Amphipoda, Phoxocephalopsidae). J Crust Biol 2: 261-272.

BARNARD JL AND CLARK J. 1984. Redescription of Phoxocephalopsis zimmeri with a new species, and establishment of the family Phoxocephalopsidae (Crustacea, Amphipoda) from magellanic South America. J Crust Biol 4: 85-105.

BARNARD JLAND DRUMMOND MM. 1979. Gammaridean Amphipoda of Australia, Part IV. Smithson Contr Zool 269: 1-69.

BARNARD JLAND DRUMMOND MM. 1982. Gammaridean Amphipoda of Australia. Part V. Superfamily Haustorioidea. Smithson Contr Zool 360: 1-148.

BARNARD JL AND KARAMAN GS. 1991. The families and genera of marine gammaridean Amphipoda (except marine gammaroids). Part 2. Rec Aust Mus 13(2): 419-866.

BARNARD JL AND THOMAS JD. 1988. Ipanemidae, new family, Ipanema talpa, new genus and species, from the surf zone of Brazil (Amphipoda: Gammaridea: Haustorioidea). Proc Biol Soc Wash 101: 614-621.

BARNARD KH. 1931. Diagnosis of new genera and species of amphipod Crustacea collected during the 'Discovery' Investigations, 1925-1927. Ann Mag Nat Hist 10(7): 425430.

BOECK A. 1871. Crustacea Amphipoda borealia et arctica. Forhandlinger i Videnskabs-Selskabet i Christiania 1870: 81-280 + i-viii.
BOOS H, BUCKUP GB, BUCKUP L, ARAUJO PB, MAGALHÃES C, ALMERÃO MP AND MANTELATTO FL. 2012. Checklist of the Crustacea from the state of Santa Catarina, Brazil. Check List 8(6): 1020-1046.

BOUSFIELD EL. 1978. A revised classification and phylogeny of amphipod crustaceans. Trans R Soc Can 4: 343-390.

BOUSFIELD EL AND SHIH CT. 1994. The phyletic classification of amphipod crustaceans: Problems in resolution. Amphipacifica 1(3): 76-134.

COLEMAN CO. 2006. Substituting time-consuming pencil drawings in arthropod taxonomy using stacks of digital photographs. Zootaxa 1360: 61-68.

CREED JC, PIRES DO AND FIGUEIREDO MAO. 2007. Biodiversidade marinha da Baía da Ilha Grande. Brasília: Ministério do Meio Ambiente, Série Biodiversidade 23, $416 \mathrm{p}$.

DANA JD. 1849. Synopsis of the genera of Gammaracea. Am J Sci Arts (Series 2) 8: 135-140.

DANA JD. 1852. On the classification of the Crustacea Choristopoda or Tetradecapoda. Am J Sci Arts (Series 2) 14: 297-316.

DE BROYER C, LOWRY JK, JAZDZEWSKI K AND ROBERT H. 2007. Catalogue of the gammaridean and corophiidean Amphipoda (Crustacea) of the Southern Ocean with distribution and ecological data. In: DE BROYER C. (Eds), Census of Antarctic Marine Life. Synopsis of the Amphipoda of the Southern Ocean. Vol. 1. Bulletin de L'Institut Royal des Sciences Naturelles de Belgique Biologie 77 (Suppl. 1), p. 1-325.

D'UDEKEM AND D'ACOZ C. 2006. Systematic, phylogenetic and biological considerations on the genera Bathyporeia, Amphiporeia, Pontoporeia and Priscillina, with redescription of the West-Atlantic Bathyporeia species and description of a new Priscillina from Svalbard (Crustacea, Amphipoda). Bull Inst R Sci Nat Belg Biologie 76: 33-110.

FALCÃO APC, CURBELO-FERNANDEZ MP, BORGES ALN, FILGUEIRAS VL, KOWSMANN RO AND MARTINS RP 2017. Importância ecológica e econômica da Bacia de Campos: ambiente transicional na margem continental do Oceano Atlântico Sudoeste. In: Falcão APC and Lavrado HP (Eds), Ambiente Bentônico: caracterização ambiental regional da Bacia de Campos, Atlântico Sudoeste, Rio de Janeiro: Elsevier, Habitats, v. 3, p. 1-13.

GAPPA JL, ALONSO DE PINA GM AND LANDONI NA. 2006. Biodiversity of benthic Amphipoda (Crustacea: Peracarida) in Southwest Atlantic between $35^{\circ} \mathrm{S}$ and $56^{\circ} \mathrm{S}$. Zootaxa 1342: 1-66.

HUGHES L AND LÖRZ A. 2013. Family placement of the enigmaticOtagia neozelanica (Chilton, 1897) Haustorioidea: Otagiidae fam. nov. (Amphipoda: Crustacea). Zootaxa 3636(3): 439-450. 
LATREILLE PA. 1816. Amphipoda. In: Nouveau Dictionaire d'histoire naturelle, appliquée auxArts, à l'Agriculture, à l'Économie rurale et domestique, à la Médecine, etc. Par unesociété de Naturalistes et d'Agriculteurs, v. 1, $2^{\text {nd }}$ ed., Paris: Deterville, p. 467-469.

LEDOYER M. 1967. Amphipodes gammariens de quelques biotopes de substrat meuble de laregion de Tulear (Republique Malgache). Etude systematique et ecologique. Ann Univ Madag 6: 47-62.

LEDOYER M. 1986. Crustacés amphipodes gammariens: Familles des Haustoriidae à Vitjazianidae. Faune Madag 59(2): 599-1112.

LOWRY J AND MYERS A. 2017. A Phylogeny and Classification of the Amphipoda with the establishment of the new order Ingolfiellida (Crustacea: Peracarida). Zootaxa 4265(1): 1-89.

NEVES FM AND BEMVENUTI CE. 2006. Spatial distribution of macrobenthic fauna on three sandy beaches from northern Rio Grande do Sul, southern Brazil. Braz J Oceanogr 54(2): 135-145.

NEVES FM AND BEMVENUTI CE. 2009. Variabilidade diária da zonação da macrofauna bentônica em praias arenosas do litoral norte do Rio Grande do Sul. Iheringia. Sér Zool 99: 71-81.

REN X. 1999. A new family of superfamily Haustoroidea (Crustacea: Amphipoda: Gammaridea) from the China Sea. Chin J Oceanol Limnol 17(4): 344-349.

RIBEIRO-FERREIRA VP ET AL. 2017. Métodos empregados na avaliação do compartimento bentônico da Bacia de Campos. In: Falcão APC and Lavrado HP (Eds), Ambiente Bentônico: caracterização ambiental regional da Bacia de Campos, Atlântico Sudoeste, Rio de Janeiro: Elsevier, Habitats, v. 3, p. 15-39.

RUFFO S. 1956. Su alcuni anfipodi raccolti sulle coste atlantische del Brasile. Mem Mus Civico Storia Nat Verona 5: 115-124.

SARS GO. 1891. Amphipoda. Part VII. Phoxocephalidae. An account of the Crustacea of Norway, with short descriptions and figures of all the species:. I: 141-160, pls 49-60. Cammermeyer, Christiana (Oslo).

SCHELLENBERG A. 1931. Gammariden und Caprelliden des Magellangebietes, Südgeorgiens undder Westantarktis. Further Zoological Results of the Swedish Antarctic Expedition 1901-1903 2: 1-290.

SITTROP DJ, SEREJO CS, SOUZA-FILHO JF, SENNA AR. 2014. New genera and species of Urothoidae (Amphipoda) from the Brazilian deep sea, with the re-assignment of Pseudurothoe and Urothopsis to Phoxocephalopsidae. J Nat Hist 49(9-10): 527-552.

STEBBING TRR. 1906. Amphipoda. I. Gammaridea. Das Tierreich 21: 1-806.

THURSTON MH. 1982. Cheus annae, new genus, new species (Cheidae, new family), a fossorial amphipod from the Falkland Islands. J Crust Biol 2: 410-419.

THURSTON MH. 1989. A new genus and species of fossorial amphipod from the Falkland Islands (Crustacea, Amphipoda, Phoxocephalopsidae), with notes on Phoxocephalopsis. J Nat Hist 23: 299-310.

WAKABARA Y AND SEREJO CS. 1998. Malacostraca Peracarida. Amphipoda. Gammaridea and Caprellidea. In: Young PS (Ed), Catalogue of Crustacea of Brazil. Série Livros 6. Rio de Janeiro: Museu Nacional, p. 561-594.

WAKABARA Y, TARARAM AS, VALÉRIO-BERARDO MT, DULEBA W AND LEITE FPP. 1991. Gammaridean and caprellidean fauna from Brazil. Hydrobiologia 223: 69-77.

WATLING L. 1989. A classification system for crustacean setae based on the homology concept. In: Felgenhauer BE, Watling L and Thistle AB (Eds), Functional Morphology of Feeding and Grooming in Crustacea. Crustacean Issues, v. 6, Balkema, Rotterdam, p. 15-27.

ZIMMER A, ARAUJO PB AND BOND-BUCKUP G. 2009. Diversity and arrangement of the cuticular structures of Hyalella (Crustacea: Amphipoda: Dogielinotidae) and their use in taxonomy. Zoologia 26(1): 127-142. 